\title{
Transmission-Line Model for Materials with Spin-Momentum Locking
}

\author{
Shehrin Sayed, ${ }^{1, *}$ Seokmin Hong, ${ }^{2}$ and Supriyo Datta ${ }^{1, \dagger}$ \\ ${ }^{1}$ School of Electrical and Computer Engineering, Purdue University, West Lafayette, Indiana 47907, USA \\ ${ }^{2}$ Center for Spintronics, Korea Institute of Science and Technology, Seoul 02792, Republic of Korea
}

(Received 15 July 2017; revised manuscript received 15 April 2018; published 20 November 2018)

\begin{abstract}
We provide a transmission-line representation for channels exhibiting spin-momentum locking (SML) that can be used for both time-dependent and steady-state transport analysis on a wide variety of materials with spin-orbit coupling such as topological insulators, Kondo insulators, transition metals, semimetals, oxide interfaces, and narrow band-gap semiconductors. This model is based on a time-dependent fourcomponent diffusion equation obtained from the Boltzmann transport equation assuming linear response and elastic scattering in the channel. We classify all electronic states in the channel into four groups $\left(U^{+}\right.$, $D^{+}, U^{-}$, and $D^{-}$) depending on the spin index [up $(U)$, down $(D)$ ] and the sign of the $x$ component of the group velocity $(+,-)$ and assign an average electrochemical potential to each of the four groups to obtain the four-component diffusion equation. For normal metal channels, the model decouples into the well-known transmission-line model for charge and a time-dependent version of the Valet-Fert equation for spin. We first show that, in the steady-state limit, our model leads to simple expressions for charge-spin interconversion in SML channels in good agreement with existing experimental data on diverse materials. We then use the full time-dependent model to study spin-charge separation in the presence of SML, a subject that has been controversial in the past. Our model shows that the charge and spin signals travel with two distinct velocities, resulting in well-known spin-charge separation, which is expected to persist even in the presence of SML. However, our model predicts that the lower velocity signal is purely spin, while the higher velocity signal is largely charge with an additional spin component proportional to the degree of SML, which has not been noted before. Finally, we note that our model can be used within standard circuit simulators such as SPICE to obtain numerical results for complex geometries.
\end{abstract}

DOI: 10.1103/PhysRevApplied.10.054044

\section{INTRODUCTION}

Transport properties in materials with spin-orbit coupling (SOC) are of great interest for potential spintronic applications, especially because of the unique spinmomentum locking (SML) observed in diverse classes of materials, e.g., topological insulators (TI) [1-9], Kondo insulators [10], transition metals [11-18], semimetals [19], oxides [20-22], and narrow band-gap semiconductors [23-26]. There has been an immense effort to model the interplay between spin and charge in such materials using time-dependent classical [27] or quantum Boltzmann equations $[28,29]$, nonequilibrium Green's functions

\footnotetext{
*ssayed@purdue.edu

$\dagger$ datta@purdue.edu
}

Published by the American Physical Society under the terms of the Creative Commons Attribution 4.0 International license. Further distribution of this work must maintain attribution to the author(s) and the published article's title, journal citation, and DOI.
[30-34], phenomenological equations coupled to magnet dynamics [35], and time-independent diffusion equations used to explain the bulk spin Hall effect [36].

In this paper, we derive a transmission-line model that can be used for both time-dependent and steady-state transport analysis on multicontact-based structures implemented with materials exhibiting SML. The model is compatible within a standard circuit simulator tool such as Simulation Program with Integrated Circuit Emphasis (SPICE), which will enable straightforward analysis of complex geometries. The proposed model is an addition to our multiphysics spin-circuit framework [37], which has been previously used to explain experiments and evaluate spin-based device proposals $[38,39]$. We first show that, in the steady-state limit, the transmission-line model provides simple expressions for charge-spin interconversion that have been previously used by a number of experimental groups $[3-10,26]$ to quantify spin voltages on diverse materials measured with ferromagnetic contacts. We further derive a simple expression for a parameter that has been widely used to quantify the inverse Rashba-Edelstein effect (IREE) in two-dimensional (2D) channels, which 
shows good agreement with existing experimental data on diverse materials [20,40-42]. We then use the full time-dependent model to study spin-charge separation in the presence of SML in materials with SOC, a subject that has been controversial in the past (see, for example, [43-46]). Our model suggests that, depending on the channel cross section, the charge and spin signals travel with two distinct velocities, resulting in spin-charge separation, which is well-known for materials without SOC [47-50] based on the Luttinger liquid theory. However, our model shows that the lower-velocity signal is purely spin, while the higher-velocity signal is largely charge, with an additional spin component proportional to the degree of SML in the channel, which has not been discussed before.

The transmission-line model is based on a timedependent four-component diffusion equation obtained from the Boltzmann transport equation assuming linear response and elastic scattering processes in the channel. The assumptions made to derive the model are discussed in detail in Sec. V. The basic approach is to assign one electrochemical potential $\mu(\vec{p}, s)$ to each of the eigenstates $(\vec{p}, s)$, where $\vec{p}$ is the momentum confined in the $z-x$ plane and $s= \pm 1$ is the spin index with +1 and -1 denoting the up $(U)$ and the down $(D)$ spins with respect to the spin quantization axis $\hat{y} \times(\vec{p}-q \vec{A})(\vec{A}$ is the vector magnetic potential). We then classify the eigenstates into four groups $\left(U^{+}, D^{+}, U^{-}\right.$, and $\left.D^{-}\right)$based on the spin index $(U$, $D)$ and the sign of the $x$ component of the group velocity $(+,-)$ and define an average electrochemical potential corresponding to each of the four groups, resulting in a four-component diffusion equation. This procedure can be viewed as an extension of the Valet-Fert equation, which uses two electrochemical potentials for $U$ and $D$ states [51].

In our generalized view, $U^{+}$(and $U^{-}$) states have the same number of modes $M$ (and $N$ ) as $D^{-}$(and $D^{+}$) states due to the time-reversal symmetry. The degree of SML in our model is given by $[52,53]$

$$
p_{0}=\frac{M-N}{M+N},
$$

where $M$ and $N$ are evaluated at the Fermi energy for zero temperature and, in general, require thermal averaging. For normal metal (NM) channels, $p_{0}=0$, i.e., $M=$ $N$. For a perfect topological insulator (TI) $N=0$ leading to $p_{0}=1$, however, $p_{0}$ gets effectively lowered by the presence of parallel channels. $p_{0}$ has been quantified for different TIs by a number of groups [3-9] by measuring the charge-current-induced spin voltage using a ferromagnetic (FM) contact, motivated by a theoretical proposal [34]. For a Rashba channel with a coupling coefficient, $\alpha_{R}$, $p_{0} \approx \alpha_{R} k_{F} /\left(2 E_{F}\right) \ll 1[25,34]$, which has been quantified with similar spin voltage measurements [26]. Here, $k_{F}$ and $E_{F}$ are the Fermi wave vector and the Fermi energy, respectively. Recently, spin voltage measurements have been reported on transition metals ( $\mathrm{Ta}, \mathrm{Pt}, \mathrm{W}, \mathrm{Ir}$, and $\mathrm{Au}$ ) [1618,54]; semimetals ( $\mathrm{WTe}_{2}$ [19]); and Kondo insulators $\left(\mathrm{SmB}_{6}[10]\right)$. These experiments can also be quantified by $p_{0}$, although the underlying mechanism of transition metals is subject to active debate [55-57] and could involve a bulk spin Hall effect $[14,36,58]$ or interface Rashba-like channel [15,59-61].

The four-component diffusion equation in steady state reduces to our prior model that predicted a unique threeresistance state in a two-FM-contact-based multiterminal spin-valve structure on SML materials [52]. The prediction has recently been observed in InAs [26] and Pt $[16,17]$ up to room temperature and should be observed in other materials with SML as well. We translate the full time-dependent version of the four-component diffusion equation into a transmission-line model with twocomponent (charge and $z$ component of spin) voltages and currents, where the coupling between the charge and spin in a SML channel is characterized by $p_{0}$ in Eq. (1). Note that the proposed model does not take into account the effects such as spin precession involving the off-diagonal elements of the density matrix, which we assume to be negligible. An extension of this model to include $x$ and $y$ components of spin could possibly address such issues, as done earlier for materials without SOC (see [38] and references therein). For NM channels (i.e., $p_{0}=0$ ), the proposed transmission-line model decouples into the wellknown model for charge that has been previously used to analyze transport in quantum wires $[50,62,63]$ and a time-dependent version of the Valet-Fert equation [51] for spin.

The paper is organized as follows. In Sec. II, we describe the transmission-line model for SML channels and show that special cases lead to prior well-known models. In Sec. III, we derive several results on charge-spin interconversion from our transmission-line model in steady state and present comparison with SPICE simulations using the full model. We obtain a simple expression for a parameter that has been widely used to quantify IREE and show that it is in good agreement with available experiments on diverse materials. In Sec. IV, we study the spin-charge separation in terms of spin and charge signal velocities obtained from our time-dependent transmission-line equations. We show that the separation persists even in SML channels; however, in SML channels there exists an additional spin component accompanied by the high-velocity charge signal. In Sec. V, we derive the transmission-line model starting from the Boltzmann transport equation with all the assumptions clearly stated. We discuss different scattering mechanisms in the channels and their effects on charge and spin transport. Finally, in Sec. VI, we end with a brief summary. 


\section{TRANSMISSION-LINE MODEL}

\section{A. Model description}

We consider the structure and axes in Fig. 1(a) to derive the transmission-line model. The model has two components: the charge and $z$ component of spin, with a coupling between them characterized by $p_{0}$ in Eq. (1). The charge model is given by

$$
\begin{aligned}
\left(\frac{1}{C_{E}}+\frac{1}{C_{Q}}\right)^{-1} \frac{\partial}{\partial t} V_{c} & =-\frac{\partial}{\partial x} I_{c}, \\
\left(L_{K}+L_{M}\right) \frac{\partial}{\partial t} I_{c}+R_{c} I_{c} & =-\frac{\partial}{\partial x} V_{c}+p_{0} \eta_{c} V_{s},
\end{aligned}
$$

where $I_{c}$ and $V_{c}$ are the charge current and voltage along the $\hat{x}$ direction, $C_{E}$ and $C_{Q}$ are the electrostatic and quantum capacitances per unit length respectively, $L_{M}$ and $L_{K}$ are the magnetic and kinetic inductances per unit length respectively, and $R_{c}$ is the charge resistance per unit length.

The spin model is given by

$$
\begin{aligned}
\frac{C_{Q}}{\alpha^{2}} \frac{\partial}{\partial t} V_{s}+G_{\mathrm{sh}} V_{s}+p_{0} g_{m} V_{\mathrm{em}} & =-\frac{\partial}{\partial x} I_{s}+p_{0} \gamma_{s} I_{c}, \\
\alpha^{2} L_{K} \frac{\partial}{\partial t} I_{s}+R_{s} I_{s}-p_{0} r_{m} I_{\mathrm{em}} & =-\frac{\partial}{\partial x} V_{s}+p_{0} \eta_{s} V_{c},
\end{aligned}
$$

where $I_{S}$ and $V_{S}$ are the spin current and voltage along the $\hat{x}$ direction with spin polarization along the $\hat{z}$ direction. In this discussion, the $\hat{y}$ direction is out of plane. Here, $\alpha=2 / \pi$ is an angular averaging factor, $R_{s}$ is the series spin resistance per unit length of the channel, and $G_{\text {sh }}$ is the shunt spin conductance per unit length that captures the spin lost in the channel due to the spin relaxation. A detailed derivation of Eqs. (2) and (3) from the Boltzmann transport equation will be discussed in Sec. V with clearly stated assumptions.

Distributed circuit models for charge and spin are shown in Figs. 1(b) and 1(c), which are based on Eqs. (2) and (3), respectively. The dependent sources proportional to $p_{0}$ represent charge-spin intercoupling between the two models. The dependent source parameters in Fig. 1 are given by

$$
\begin{gathered}
V_{m}=\eta_{c} V_{s}, \\
V_{n}=\eta_{s} V_{c}+r_{m} I_{\mathrm{em}}, \\
\text { and, } I_{n}=\gamma_{s} I_{c}-g_{m} V_{\mathrm{em}} .
\end{gathered}
$$

The parameters of Eqs. (2) and (3) are given by

$$
C_{Q}=\frac{2}{R_{B}\left|\left\langle v_{x}\right\rangle\right|},
$$

(a) Structure

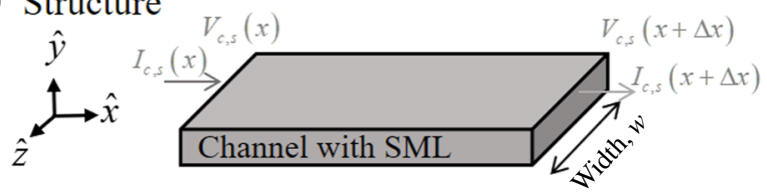

(b) Charge transmission line

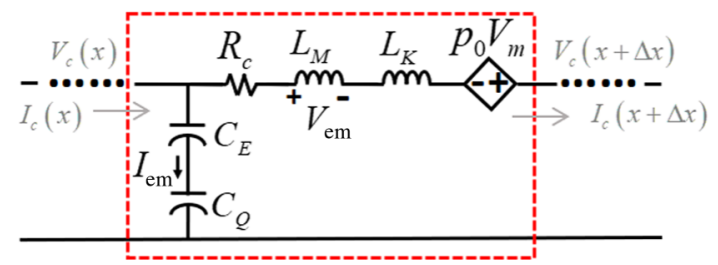

(c) Spin transmission line

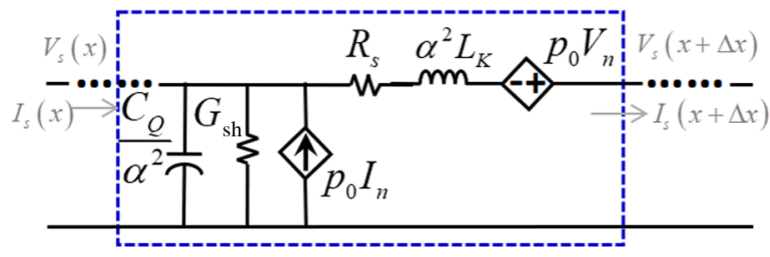

FIG. 1. (a) Structure and corresponding axes of the channel with spin-momentum locking, for which a transmission-line model is derived. The model has two components: (b) charge [which corrseponds to Eq. (2)] and (c) spin [which corrseponds to Eq. (3)], with a coupling between them described by the degree of SML $p_{0}$ [see Eq. (1)]. The coupling between the charge and spin is modeled by dependent voltage and current sources with $V_{m}=\eta_{c} V_{s}, V_{n}=\eta_{s} V_{c}+r_{m} I_{\mathrm{em}}$, and $I_{n}=\gamma_{s} I_{c}-g_{m} V_{\mathrm{em}}$.

$$
\begin{gathered}
L_{K}=\frac{R_{B}}{2\left|\left\langle v_{x}\right\rangle\right|}, \\
R_{c}=\frac{R_{B}}{\lambda}, \\
R_{s}=\frac{\alpha^{2} R_{B}}{\lambda_{0}},
\end{gathered}
$$

$$
\begin{gathered}
G_{\mathrm{sh}}=\frac{4}{\alpha^{2} R_{B} \lambda_{s}}, \\
g_{m}=\frac{2}{\alpha R_{B}},
\end{gathered}
$$

$$
r_{m}=\frac{\alpha}{\left|\left\langle v_{x}\right\rangle\right| C_{E}},
$$

$$
\eta_{s}=\frac{2 \alpha}{\lambda_{0}},
$$




$$
\begin{aligned}
\eta_{c} & =\frac{2}{\alpha \lambda_{r}}, \\
\gamma_{s} & =\frac{2}{\alpha \lambda_{t}}, \\
\text { and } \quad R_{B} & =\frac{h}{q^{2}} \frac{1}{M+N} .
\end{aligned}
$$

Here, $\lambda, \lambda_{0}$, and $\lambda_{s}$ are the three distinct mean free paths that determine $R_{c}, R_{s}$, and $G_{\mathrm{sh}}$, respectively. $\left|\left\langle v_{x}\right\rangle\right|$ is the magnitude of the thermally averaged electron velocity $\left\langle v_{x}\right\rangle, \eta_{c}$ represents the spin-to-charge conversion coefficient and $\eta_{s}, \gamma_{s}$ represent charge-to-spin conversion coefficients. These coefficients depend on different scattering mechanisms in the channel, which will be discussed later in Sec. V. $r_{m}$ and $g_{m}$ are transient charge-spin coupling coefficients, which are in the units of resistance per unit length and conductance per unit length, respectively.

$R_{B}$ is the ballistic resistance of the channel, $h$ is the Planck's constant, and $q$ is the electron charge. $R_{B}$ is inversely proportional to the total number of modes $(M+$ $N$ ) in the channel, which represents a material property and does not imply ballistic transport. The models and related results discussed in this paper are valid all the way from the ballistic to the diffusive regime of operation.

\section{B. Presence of an external contact}

In the presence of an external contact on the channel [see Fig. 2(a)], the charge model in Eq. (2) is modified as

$$
\begin{aligned}
\left(\frac{1}{C_{E}}+\frac{1}{C_{Q}}\right)^{-1} \frac{\partial}{\partial t} V_{c} & =-\frac{\partial}{\partial x} I_{c}+i^{c}, \\
\left(L_{K}+L_{M}\right) \frac{\partial}{\partial t} I_{c}+R_{c} I_{c} & =-\frac{\partial}{\partial x} V_{c}+p_{0} \eta_{c} V_{s}+\Delta v^{c},
\end{aligned}
$$

and the spin model in Eq. (3) is modified as

$$
\begin{aligned}
\frac{C_{Q}}{\alpha^{2}} \frac{\partial}{\partial t} V_{s}+G_{\mathrm{sh}} V_{s}+p_{0} g_{m} V_{\mathrm{em}} & =-\frac{\partial}{\partial x} I_{s}+p_{0} \gamma_{s} I_{c}+i^{s}, \\
\alpha^{2} L_{K} \frac{\partial}{\partial t} I_{s}+R_{s} I_{s}-p_{0} r_{m} I_{\mathrm{em}} & =-\frac{\partial}{\partial x} V_{s}+p_{0} \eta_{s} V_{c}+\Delta v^{s},
\end{aligned}
$$

where $i^{c}$ and $i^{s}$ represent charge and spin currents entering into the channel per unit length from the external contact. $\Delta v^{c}$ and $\Delta v^{s}$ represent the change in channel charge and spin voltages per unit length in the region under the external contact. They are given as

$$
\begin{aligned}
&\left\{\begin{array}{c}
i^{c} \\
i^{s}
\end{array}\right\}=G_{0}\left[\begin{array}{cc}
1 & \frac{p_{f}}{\alpha} \\
\frac{p_{f}}{\alpha} & \frac{1}{\alpha^{2}}
\end{array}\right]\left\{\begin{array}{l}
v_{c}-V_{c} \\
v_{s}-V_{s}
\end{array}\right\}, \quad \text { and } \\
&\left\{\begin{array}{c}
\Delta v^{c} \\
\Delta v^{s}
\end{array}\right\}=-\frac{G_{0} R_{B}^{2}}{4}\left[\begin{array}{cc}
1 & \alpha p_{f} \\
\alpha p_{f} & \alpha^{2}
\end{array}\right]\left\{\begin{array}{l}
I_{c} \\
I_{s}
\end{array}\right\} \\
&+\frac{p_{0} G_{0} R_{B}}{2}\left[\begin{array}{cc}
p_{f} & \frac{1}{\alpha} \\
\alpha & p_{f}
\end{array}\right]\left\{\begin{array}{l}
v_{c} \\
v_{s}
\end{array}\right\},
\end{aligned}
$$

where $v_{c}=\left(v_{u}+v_{d}\right) / 2$ and $v_{s}=\alpha\left(v_{u}-v_{d}\right) / 2$ are charge and spin voltages applied at the external contact, $G_{0}$ is the contact conductance per unit length, and $p_{f}$ is the contact polarization with $p_{f}=0$ indicating a normal metal contact and $p_{f} \neq 0$ indicating a ferromagnetic contact. The derivation of the model starting from the Boltzmann transport equation is shown in Sec. V with clearly stated assumptions streamlined with subheadings.

Modified distributed circuit models for charge and spin are shown in Figs. 2(b) and 2(c), which are based on Eqs. (6) and (7), respectively. The presence of a contact with conductance $G_{0}$ adds series resistances $R_{\text {cont }}^{c}=$ $G_{0} R_{B}^{2} / 4$ and $R_{\text {cont }}^{s}=\alpha^{2} G_{0} R_{B}^{2} / 4$ in the charge and spin models as shown in Fig. 2. This effect exists even if the channel is NM. The presence of the external contact also modulates the dependent sources in Eqs. (4a) and (4b) as

$$
\begin{gathered}
V_{m}^{\prime}=\eta_{c} V_{s}+\frac{G_{0} R_{B}}{2}\left(\frac{v_{s}}{\alpha}+p_{f} v_{c}\right), \\
V_{n}^{\prime}=\eta_{s} V_{c}+r_{m} I_{\mathrm{em}}+\frac{G_{0} R_{B}}{2}\left(\alpha v_{c}+p_{f} v_{s}\right),
\end{gathered}
$$

with $V_{a}$ and $V_{b}$ representing the voltage drop across $R_{\text {cont }}^{c}$ and $R_{\text {cont }}^{s}$ in charge and spin models in Fig. 2, respectively. Note that the additional terms due to the presence of an external contact, are proportional to $G_{0}$ and negligible for potentiometric contacts where $G_{0}$ is very low.

\section{Special case: Normal metals $\left(p_{0}=0\right)$}

We consider a special case of Eqs. (2) and (3) for a normal metal channel, i.e., $p_{0}=0$. For a NM channel, the charge and spin model decouples to well-known models as described below.

\section{Charge model: Transport model for quantum wires}

For NM channels $\left(p_{0}=0\right)$, the charge model in Eq. (2) reduces to the well-known transmission-line model for a 
(a) Structure

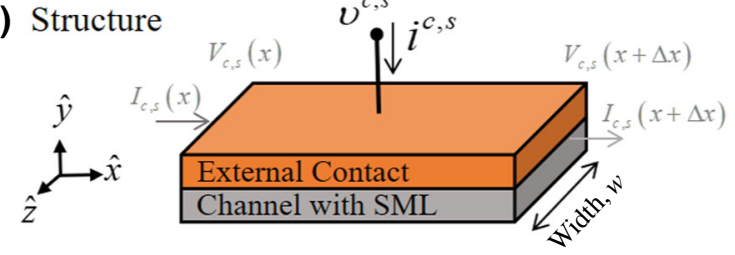

(b) Charge transmission line

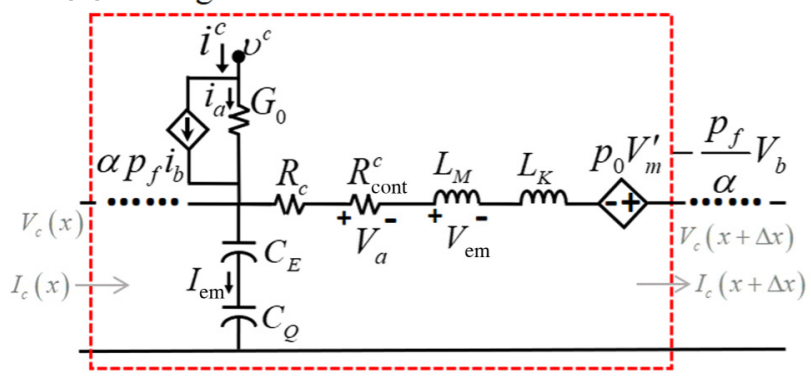

(c) Spin transmission line

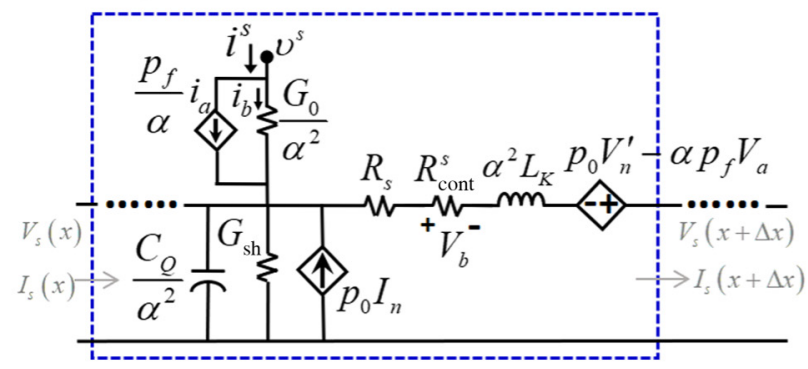

FIG. 2. (a) Structure of the spin-momentum-locked channel in the presence of an external contact. Both (b) charge and (c) spin transmission-line models are modified as compared to Fig. 1, which now correspond to Eqs. (6) and (7), respectively. The dependent sources are $V_{m}^{\prime}=\eta_{c} V_{s}+\left(G_{0} R_{B} / 2\right)\left(v_{s} / \alpha+p_{f} v_{c}\right)$, $V_{n}^{\prime}=\eta_{s} V_{c}+r_{m} I_{\mathrm{em}}+\left(G_{0} R_{B} / 2\right)\left(\alpha v_{c}+p_{f} v_{s}\right)$, and $I_{n}=\gamma_{s} I_{c}-$ $g_{m} V_{\mathrm{em}}$. Note that the model reduces to that shown in Fig. 1 in the limit $G_{0} \rightarrow 0$.

charge that has been previously used to analyze transport in quantum wires, given by

$$
\begin{aligned}
& \left(\frac{1}{C_{E}}+\frac{1}{C_{Q}}\right)^{-1} \frac{\partial}{\partial t} V_{c}=-\frac{\partial}{\partial x} I_{c}, \\
& \left(L_{K}+L_{M}\right) \frac{\partial}{\partial t} I_{c}+R_{c} I_{c}=-\frac{\partial}{\partial x} V_{c} .
\end{aligned}
$$

The model in Eq. (11) was first derived from Luttinger liquid theory $[50,62]$ and then from the Boltzmann transport equation with one electrochemical potential [63]. In the quantum wire limit, $L_{K} \gg L_{M}$ and $C_{Q} \ll C_{E}$, while, in the classical transmission-line limit, $L_{K} \ll L_{M}$ and $C_{Q} \gg C_{E}$ $[50,63]$.

In steady state $(\partial / \partial t \rightarrow 0)$, we get the diffusion equation for charge from Eq. (11), given by

$$
\frac{d^{2}}{d x^{2}} V_{c}=0
$$

\section{Spin model: Valet-Fert equation}

For NM channels $\left(p_{0}=0\right)$, the spin model in Eq. (3) becomes a time-dependent spin-diffusion equation, given by

$$
\begin{aligned}
\frac{C_{Q}}{\alpha^{2}} \frac{\partial}{\partial t} V_{s}+G_{\mathrm{sh}} V_{s} & =-\frac{\partial}{\partial x} I_{s}, \\
\alpha^{2} L_{K} \frac{\partial}{\partial t} I_{s}+R_{s} I_{s} & =-\frac{\partial}{\partial x} V_{s},
\end{aligned}
$$

which, in steady state $(\partial / \partial t \rightarrow 0)$, becomes the wellknown Valet-Fert equation [51], given by

$$
\frac{d^{2}}{d x^{2}} V_{s}=\frac{V_{s}}{\lambda_{s f}^{2}}
$$

with the spin diffusion length given by

$$
\lambda_{s f}=\frac{1}{\sqrt{R_{s} G_{\mathrm{sh}}}}=\frac{\sqrt{\lambda_{0} \lambda_{s}}}{2} .
$$

A spin model similar to Eq. (13) has been discussed previously based on Luttinger liquid theory [50]; however, the model did not take into account the spin relaxation processes in the channel (the shunt conductance $G_{\mathrm{sh}}$ ).

\section{STEADY-STATE TRANSPORT RESULTS}

In this section, we discuss several established steadystate results on charge-spin interconversion in the potentiometric limit. We start from the steady-state $(\partial / \partial t \rightarrow 0)$ form of the transmission-line model with external contact in Eqs. (6) and (7), given by

$$
\frac{d}{d x} I_{c}=i^{c}
$$

$$
\frac{d}{d x} V_{c}=-R_{c} I_{c}+p_{0} \eta_{c} V_{s}+\Delta v^{c}
$$

$$
\frac{d}{d x} I_{s}=-G_{\mathrm{sh}} V_{s}+p_{0} \gamma_{s} I_{c}+i^{s}
$$

$$
\text { and } \frac{d}{d x} V_{s}=-R_{s} I_{s}+p_{0} \eta_{s} V_{c}+\Delta v^{s} .
$$

Under the steady-state condition $(\partial / \partial t \rightarrow 0)$, the capacitors and inductors in Fig. 2 become open and short circuits, respectively. The steady-state form in Eq. (16) is equivalent to our prior time-independent semiclassical equations with four electrochemical potentials [52] and all our previous results can be reproduced using Eq. (16).

We first derive a resistance matrix for a three-terminal setup (two charge and one spin) with potentiometric contacts (see Fig. 3) and assuming reflection with spin flip to 
be the dominant scattering mechanism in the channel. We present dc simulation results on charge-spin interconversion in the SML channel using the full model [Eqs. (6) and (7)] in SPICE and compare with the results from the resistance matrix. We then derive a simple expression for a parameter that has been widely used to quantify the IREE in $2 \mathrm{D}$ channels. We compare the expression with available experiments on diverse materials as well as dc SPICE simulation results using the full model.

\section{A. Resistance matrix for potentiometric setup}

We consider a structure with three NM contacts $\left(p_{f}=0\right)$ on top of a SML channel, as shown in Fig. 3. Contacts 1 and 2 are the charge terminals and contact 3 is the spin terminal with no charge current flowing through it (i.e., $i^{c}=0$ ). We start from Eq. (16) and make two assumptions to drive the resistance matrix: (i) the contacts are potentiometric, i.e., the contact conductance per unit length $G_{0}$ is very low such that the following condition is satisfied:

$$
\frac{1}{\lambda}, \frac{1}{\lambda_{0}}, \frac{1}{\lambda_{r}} \gg \frac{G_{0} R_{B}}{4}
$$

(a)
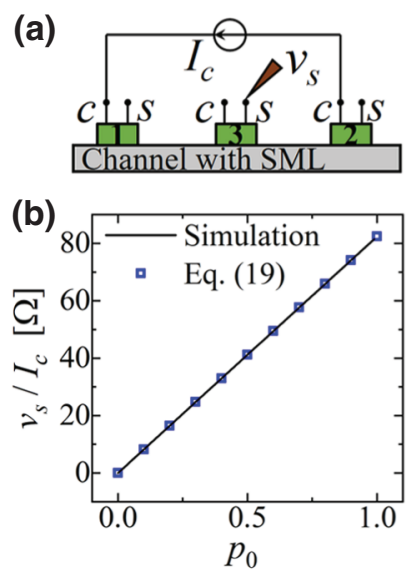

(c)
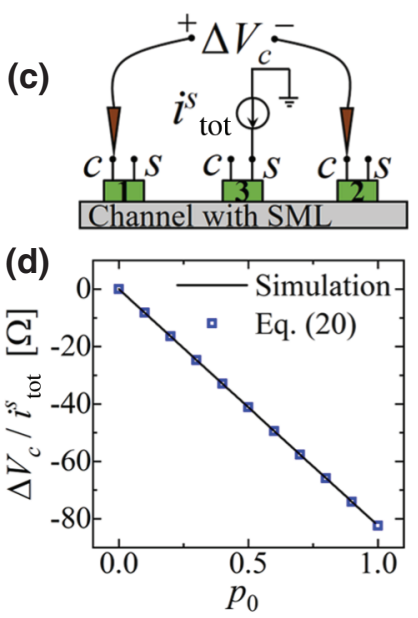

FIG. 3. (a) Setup to observe the charge-current $-I_{c}$-induced spin voltage $v_{s}$ in the SML channel. The charge terminal of contact 3 is kept open and spin terminals of contacts 1 and 2 are grounded. (b) $v_{s} / I_{c}$ vs $p_{0}$ for $i_{\text {tot }}^{s}=0$ from SPICE simulation and comparison with Eq. (19). (c) Setup to observe the spincurrent $i_{\text {tot }}^{s}$-induced charge voltage difference $\Delta V_{c}$ across the SML channel. The charge terminal of contact 3 is kept open and spin terminals of contacts 1 and 3 are grounded. (d) $\Delta V_{c} / i_{\text {tot }}^{s}$ vs $p_{0}$ for $I_{c}=0$ from SPICE simulation and comparison with Eq. (20). The SPICE setup is shown in Fig. 6 of Appendix B. The parameters are $\alpha=2 / \pi, G_{0} \approx 0.05 G_{B}, M+N=100$ and the scattering rate per unit mode $=0.04$ per lattice points. We assume reflection with spin flip to be the dominant scattering process in the channel. and (ii) the reflection with spin flip is the dominant scattering mechanism in the channel. The details of the derivation are given in Appendix A.

The resistance matrix is given by

$$
\left\{\begin{array}{c}
\Delta V_{c} \\
v_{s}
\end{array}\right\}=\left[\begin{array}{cc}
\frac{R_{B} L}{\lambda}+\frac{2}{G_{0}^{\prime \prime}} & -\frac{\alpha p_{0} R_{B}}{2} \\
\frac{\alpha p_{0} R_{B}}{2} & \frac{\alpha^{2}}{G_{0}^{\prime}}
\end{array}\right]\left\{\begin{array}{c}
I_{c} \\
i_{\text {tot }}^{s}
\end{array}\right\},
$$

where $\Delta V_{c}$ is the charge voltage difference between contacts 1 and $2, I_{c}$ is the charge current flowing in the channel with length $L, v_{s}$ is the spin voltage at contact $3, i_{\text {tot }}^{s}=i^{s} L$ is the spin current at contact 3 , contacts 1 and 2 have equal conductance $G_{0}^{\prime \prime}$, and $G_{0}^{\prime}=G_{0} L$ is the contact conductance of contact 3. Equation (18) is similar to that previously reported in Ref. [53] with corrections in the diagonal components. The diagonal components $(1,1)$ and $(2,2)$ in the matrix represent the charge resistance between contacts 1 and 2 and spin resistance at contact 3, respectively.

Note that Eq. (18) is derived under the assumption that reflection with spin flip is the dominant scattering mechanism. In the presence of other scattering mechanisms, the basic structure of Eq. (18) remains the same; however, additional factors related to scattering rates multiply each of the components in the matrix (see Appendix A).

\section{B. Direct effects: Charge-current-induced spin voltage}

For a charge current $I_{c}$ flowing through the SML channel, the open circuit spin voltage $v_{s}$ at the contact 3 (i.e., $i_{\text {tot }}^{s}=0$ ) can be derived from Eq. (18) as

$$
v_{s}=\frac{\alpha p_{0}}{2 G_{B}} I_{c} .
$$

Equation (19) was originally proposed in Ref. [34], which was later confirmed by a number of experiments [3-9] on different TI materials using potentiometric ferromagnetic contact. Recently, Eq. (19) has been used to quantify such experiments on semiconductors [26] and Kondo insulators [10]. Moreover, the charge-current-induced spin voltage has been measured on transition metals [16-18] and can be quantified by Eq. (19) as well. Note that the spin voltage measured using a contact with higher conductance will be lower than Eq. (19) due to the current-shunting effect in the contact [52]. The full model in Eqs. (6) and (7) takes into account such effects related to the contact conductances.

We simulate the structure in Fig. 3(a) by connecting the full two-component circuit models given in Figs. 1 and 2 in a distributed manner using the standard circuit rules. The details of the simulation setup are discussed in Appendix B. The simulation results of $v_{s} / I_{c}$ as a function of $p_{0}$ are shown in Fig. 3(b), which is in good agreement with Eq. (19). 


\section{Inverse effects: Spin-current-induced charge voltage}

The reciprocal effect of Eq. (19) is the spin-current- $i_{\text {tot }}^{s}{ }^{-}$ induced open-circuit charge voltage difference $\Delta V_{c}$ across the SML channel [53]. For $I_{c}=0$, we have from Eq. (18)

$$
\Delta V_{c}=-\frac{\alpha p_{0}}{2 G_{B}} i_{\text {tot }}^{s} .
$$

The reciprocal relation between Eqs. (19) and (20) including the negative sign has been observed experimentally $[6,17,26]$. Note that Eq. (20) is exact for a potentiometric contact. For a contact with higher conductance, current shunting effect will lower $\Delta V_{c}$ than Eq. (20) by the same amount as that for the direct effect [in Eq. (19)] [52], which can be analyzed using the full model in Eqs. (6) and (7). We simulate the structure shown in Fig. 3(c) using the full twocomponent models in Figs. 1 and 2. The simulation results of $\Delta V_{c} / i_{\text {tot }}^{s}$ as a function of $p_{0}$ are shown in Fig. 3(d), which show good agreement with Eq. (20). The details of the simulation setup are discussed in Appendix B.

\section{IREE length}

The phenomenon described by Eq. (20) is often known as the inverse Rashba Edelstein effect for 2D channels. IREE is often quantified with a parameter called IREE length, defined as

$$
\lambda_{\mathrm{IREE}}=\frac{J_{c}}{J_{s}},
$$

where $J_{c}$ is the longitudinal short-circuit charge current density in $\mathrm{A} / \mathrm{m}$ induced by the injected transverse spin current density $J_{s}$ in $\mathrm{A} / \mathrm{m}^{2}$.

We derive a simple expression for IREE length starting from the first row of Eq. (18) for a short-circuit condition between contacts 1 and 2 (i.e., $v_{c 1}=v_{c 2}$ ) and assuming large channel resistance compared to the contact resistance [i.e., $\left.L /\left(G_{B} \lambda\right) \gg 2 / G_{0}^{\prime \prime}\right]$. The expression is given by

$$
\lambda_{\text {IREE }}=\frac{\alpha p_{0} \lambda}{2}
$$

The derivation is given in Appendix A. Note that both $p_{0}$ and $\lambda$ are two completely independent parameters.

Here, $\alpha$ is an angular averaging factor that can vary between 0 and 1 depending on the angular variation of the spin polarization of the eigenstates and details of the scattering mechanism. We assume that the distribution is such that the angle between the $z$ axis and the spin polarization of the eigenstates with a particular group velocity $(+$ or - ) varies between $-\pi / 2$ to $+\pi / 2$, which yields $\alpha=2 / \pi$.
Thus, from Eq. (22), we have the following expression:

$$
\lambda_{\text {IREE }}=\frac{p_{0} \lambda}{\pi},
$$

which has been previously reported in Ref. [52]. We simulate the setup in Fig. 4(a) in order to estimate $\lambda_{\text {IREE }}$ of diverse ranges of $p_{0}$ and $\lambda$. The setup in Fig. 4(a) is same as that in Fig. 3(c), except we observe the short-circuit charge current $I_{c}$ between the charge terminals of contacts 1 and 2 induced by the injected spin current $i_{s}$ through the spin terminal of contact 3 .

We compare the simulation results with Eq. (23) as well as available measurements from spin-pumping and lateral spin valve experiments on different Rashba interfaces and a TI channel: $\mathrm{Ag} / \mathrm{Bi}$ [40], $\mathrm{Cu} / \mathrm{Bi}$ [41], $\mathrm{LaAlO}_{3} / \mathrm{SrTiO}_{3}$ (LAO/STO) [20], and $\mathrm{Bi}_{2} \mathrm{Se}_{3}$ [42]. The comparison is shown in Fig. 4(b). We estimate $\lambda$ from the reported
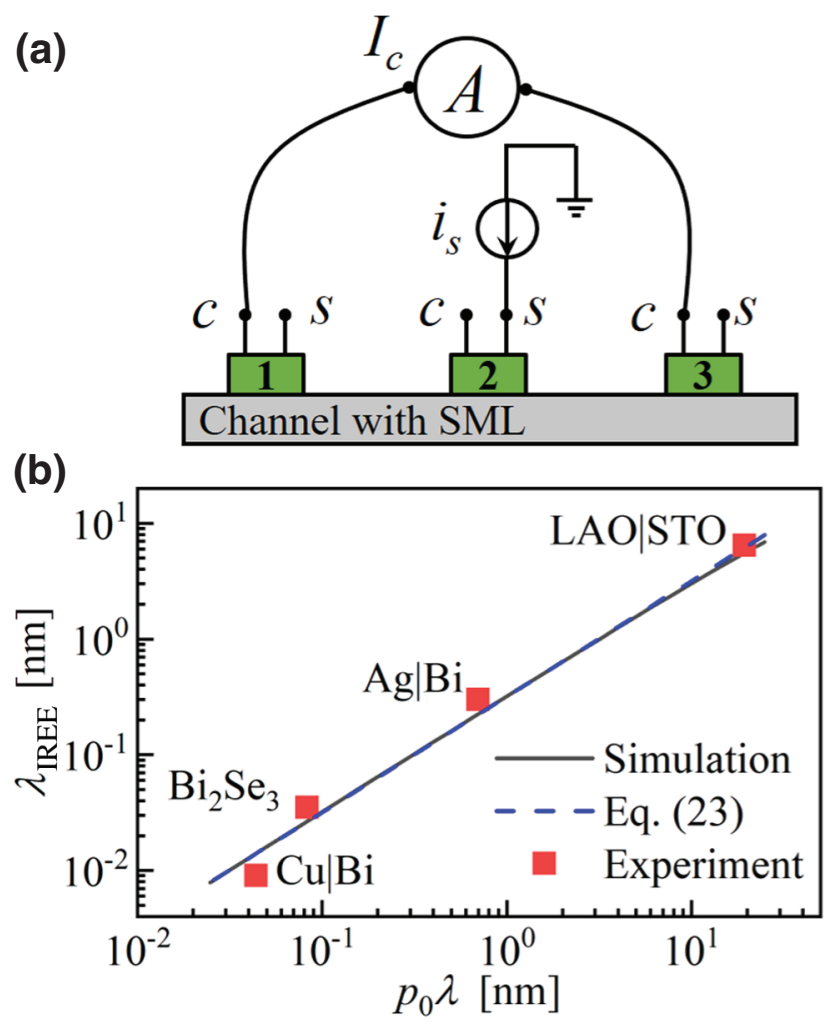

FIG. 4. (a) Setup similar to that in Fig. 3(c) but short-circuit charge current between contacts 1 and 2 is being observed. (b) IREE length $\left(\lambda_{\text {IREE }}\right)$ vs $p_{0} \lambda$ from SPICE simulation and comparison to Eq. (23) and experiments on different interfaces with Rashba SOC: Ag/Bi [40], $\mathrm{Cu} / \mathrm{Bi}$ [41], $\mathrm{LaAlO}_{3} / \mathrm{SrTiO}_{3}$ (LAO/STO) [20], and a TI channel $\mathrm{Bi}_{2} \mathrm{Se}_{3}$ [42]. The backscattering length $\lambda$ is estimated from measured sheet resistance or resistivity. The degree of spin-momentum locking $p_{0}$ is estimated from the Rashba coupling coefficient and the Fermi velocity using Eq. (24). The details of estimations are given in Appendix B. The SPICE simulation parameters are $\alpha=2 / \pi$, $G_{0} \approx 0.05 G_{B}$, and $M+N=100$. We assume the reflection with spin flip to be the dominant scattering process in the channel. 
TABLE I. Estimated material parameters. The estimations are discussed in detail in Appendix C.

\begin{tabular}{lcccc}
\hline \hline Material & $\lambda(\mathrm{nm})$ & $p_{0}$ & $\begin{array}{c}\lambda_{\text {IREE }}(\mathrm{nm}) \\
\text { [Eq. (23)] }\end{array}$ & $\begin{array}{c}\lambda_{\text {IREE }}(\mathrm{nm}) \\
\text { (measured) }\end{array}$ \\
\hline $\mathrm{Ag} / \mathrm{Bi}$ & $22.6^{\mathrm{a}}$ & $0.05^{\mathrm{b}}$ & 0.36 & $0.3[40]$ \\
$\mathrm{Cu} / \mathrm{Bi}$ & $0.88^{\mathrm{a}}$ & $0.05^{\mathrm{b}}$ & 0.014 & $0.009[41]$ \\
$\mathrm{LAO} / \mathrm{STO}$ & $180.8^{\mathrm{a}}$ & $0.0616^{\mathrm{b}}$ & 3.55 & $6.4[20]$ \\
$\mathrm{Bi}_{2} \mathrm{Se}_{3}$ & $3.2^{\mathrm{a}}$ & $0.025^{\mathrm{c}}$ & 0.026 & $0.035[42]$ \\
\hline \hline
\end{tabular}

${ }^{a}$ Estimated from the measured sheet resistance or resistivity of the samples.

${ }^{b}$ Estimated from the Rashba coupling coefficient and Fermi velocity of the materials.

${ }^{c}$ Estimated from spin-pumping-induced voltage and Eq. (20).

sheet resistance or resistivity of the samples. $p_{0}$ for $\mathrm{Ag} / \mathrm{Bi}, \mathrm{Cu} / \mathrm{Bi}$, and $\mathrm{LAO} / \mathrm{STO}$ are estimated using the Rashba coupling coefficient $\left(v_{0}\right)$ and the Fermi velocity $\left(v_{F}\right)$ quoted in the literature, using the following expression:

$$
p_{0}=\frac{v_{0}}{\sqrt{v_{0}^{2}+v_{F}^{2}}} .
$$

For $\mathrm{Bi}_{2} \mathrm{Se}_{3}$, we estimate $p_{0}$ from spin-pumping-induced inverse voltage and Eq. (20). Note that $p_{0}$ estimated for the sample in Ref. [42] is much lower than previous reports [3-9], which may be due to the presence of a large number of parallel channels. We expect higher $\lambda_{\text {IREE }}$ for $\mathrm{Bi}_{2} \mathrm{Se}_{3}$ samples with higher $p_{0}$.

The derivation of Eq. (24) from the Rashba Hamiltonian is shown in Appendix C. The estimations are summarized in Table I and the details are given in Appendix C. These two independent estimations of $p_{0}$ and $\lambda$ when applied to Eq. (23) agree very well with experimentally reported $\lambda_{\text {IREE }}$, as shown in Fig. 4 and Table I.

\section{TIME-DEPENDENT TRANSPORT RESULTS}

In this section, we use the full time-dependent model in Eqs. (2) and (3) to discuss a well-known phenomenon called the spin-charge separation. The spin-charge separation in the presence of spin-orbit coupling is a subject that has been controversial in the past. Our model shows that the charge and spin propagates with two distinct velocities that persist even in the materials with spin-orbit coupling exhibiting spin-momentum locking $\left(p_{0} \neq 0\right)$. However, we show that the lower-velocity signal is purely spin, while the higher-velocity signal is largely charge with an additional spin component proportional to $p_{0}$.

\section{A. Velocities of charge and spin signals}

The velocities for charge and spin signals can be derived by finding the eigenvalues of Eqs. (2) and (3) assuming the low loss limit and constant coefficients. In addition, we find the corresponding eigenvectors to analyze the coupling between spin and charge in the channel due to SML. The details of the derivation are given in Appendix D.

The lower-velocity eigenvalue is given by

$$
v_{g, s}= \pm \frac{1}{\sqrt{L_{K} C_{Q}}}= \pm\left\langle v_{x}\right\rangle
$$

which is determined by the quantum capacitance $C_{Q}$ and the kinetic inductance $L_{K}$, resulting in thermally averaged electron velocity $\left\langle v_{x}\right\rangle$. The corresponding eigenvector is given by

$$
\left\{\begin{array}{l}
\tilde{V}_{c} \\
\tilde{I}_{c}
\end{array}\right\}=\left\{\begin{array}{l}
0 \\
0
\end{array}\right\} \quad \text { and } \quad\left\{\begin{array}{l}
\tilde{V}_{s} \\
\tilde{I}_{s}
\end{array}\right\}=\left\{\begin{array}{c} 
\pm \alpha^{2} \sqrt{\frac{L_{K}}{C_{Q}}} \\
1
\end{array}\right\} \text {, }
$$

which shows that the lower-velocity signal is purely spin and no charge accompanies the signal even in channels with SML, i.e., $p_{0} \neq 0$.

The higher-velocity eigenvalue is given by

$$
v_{g, c}= \pm \frac{1}{\sqrt{L_{\mathrm{eff}} C_{\mathrm{eff}}}}
$$

where $C_{\mathrm{eff}}$ is a series combination of $C_{E}$ and $C_{Q}$ and $L_{\mathrm{eff}}$ is a series combination of $L_{M}$ and $L_{K}$. The corresponding eigenvector is given by

$$
\begin{aligned}
& \left\{\begin{array}{l}
\tilde{V}_{c} \\
\tilde{I}_{c}
\end{array}\right\}=\left\{\begin{array}{c} 
\pm \sqrt{\frac{L_{\mathrm{eff}}}{C_{\mathrm{eff}}}} \\
1
\end{array}\right\} \text { and } \\
& \left\{\begin{array}{l}
\tilde{V}_{s} \\
\tilde{I}_{s}
\end{array}\right\}=\frac{p_{0}}{v_{g, c}^{2}-v_{g, s}^{2}}\left\{\begin{array}{c}
-\alpha^{2} g_{m} \frac{L_{M}}{C_{Q}} v_{g, c}^{2}+\frac{r_{m}}{\alpha^{2}} v_{g, s}^{2} \\
\pm v_{g, c} v_{g, s}^{2} C_{Q}\left(-\alpha^{2} g_{m} \frac{L_{M}}{C_{Q}}+\frac{r_{m}}{\alpha^{2}}\right)
\end{array}\right\},
\end{aligned}
$$

which shows that the higher-velocity signal is largely charge that will be accompanying an additional spin signal proportional to $p_{0}$, which has not been discussed before. This additional spin component vanishes in a NM channel where there is no SML (i.e., $p_{0}=0$ ) and the signal is purely charge. Further evaluation of this high-velocity spin component we leave to future work.

The quantum capacitance $C_{Q}$ is proportional to the total number of modes $(M+N)$ in the channel [see Eq. (5a)], while the kinetic inductance $L_{K}$ is inversely proportional to $M+N$ [see Eq. (5b)]. $M+N$ is proportional to the channel width (for two dimensions) or cross-sectional area (for three dimensions) [64].

For a conductor with a very large cross section, we may have $C_{E} \ll C_{Q}$ and $L_{M} \gg L_{K}$, which is the standard transmission-line limit. In this limit, the velocity in 
Eq. (27) becomes

$$
c=\frac{1}{\sqrt{L_{M} C_{E}}},
$$

which is the velocity predicted by standard transmissionline theory and can be as high as the speed of light.

For a conductor with a very small cross section, such as quantum wires, we may have $C_{E} \gg C_{Q}$ and $L_{M} \ll L_{K}$. In this limit, the velocity in Eq. (27) becomes the thermally averaged electron velocity, given by

$$
\left\langle v_{x}\right\rangle=\frac{1}{\sqrt{L_{K} C_{Q}}},
$$

which is the same as Eq. (25). Note that the two velocity eigenvalues are equal at this limit.

\section{B. Spin-charge separation}

From Eqs. (25) and (27), we have

$$
\frac{v_{g, s}}{v_{g, c}}=\sqrt{\frac{1+\delta \frac{C_{Q}}{C_{E}}}{1+\frac{C_{Q}}{C_{E}}}}
$$

where $\delta=\left(L_{M} C_{E}\right) /\left(L_{K} C_{Q}\right)=\left(\left\langle v_{x}\right\rangle / c\right)^{2}$ [see Eq. (6) of Ref. [63]], which is usually much less than 1 , making the spin signal slower than the charge signal, given by

$$
v_{g, s}<v_{g, c} .
$$

This process results in spin-charge separation, which is well established for channels without SML (i.e., $p_{0}=$ 0 ) from Luttinger liquid theory (see, for example, Refs. [47-50], and references therein). Electrons' charge excites $C_{E}$ and $L_{M}$; hence, the charge signal velocity is determined by $C_{\text {eff }}$ and $L_{\text {eff }}$ given by Eq. (27). However, pure spin signals do not excite $C_{E}$ and $L_{M}$; hence, its velocity is determined by $C_{Q}$ and $L_{K}$ only, given by Eq. (25). Similar arguments have been made in the past $[50,62]$ in the context of carbon nanotubes without SOC.

Note that the argument in Eq. (30) is independent of $p_{0}$ (see Appendix D), which indicates that the spin-charge separation persists even in channels with SOC exhibiting SML (i.e., $p_{0} \neq 0$ ). Similar arguments have been discussed previously by considering SOC [43-45], although there exists arguments that the presence of SOC may destroy the spin-charge separation [46].

In SML channels, an additional spin signal proportional to $p_{0}$ accompanies the charge signal at the same velocity as the charge $\left(v_{g, c}\right)$ as seen from Eq. (28). This additional spin component is induced by the instantaneous voltage drop across $L_{M}$ and the instantaneous current through $C_{E}$ of the channel. However, the low-velocity signal [see Eq. (25)] remains purely spin since the spin signals do not excite $L_{M}$ and $C_{E}[50,63]$ to induce a similar accompanying charge component.

\section{TRANSMISSION-LINE MODEL FROM BOLTZMANN FORMALISM}

In this section, we derive the transmission-line model in Eqs. (6) and (7) starting from the time-dependent Boltzmann transport equation under several assumptions. The assumptions allowed us to obtain the simple expressions for the model parameters stated in Eq. (5). Several of our predictions for steady-state transport [52] have already received experimental support $[16,17,26]$ suggesting that the assumptions used here are in the reasonable limit. The assumptions can be revisited as the field evolves, which may effectively change the model parameters; however, the general structure of our model [Eqs. (6) and (7)] should remain valid.

\section{A. Boltzmann transport equation}

We assume a structure where the spatial variations and the applied fields are along the $\hat{x}$ direction. The timedependent Boltzmann transport equation is given by

$$
\frac{\partial f}{\partial t}+v_{x} \frac{\partial f}{\partial x}+F_{x} \frac{\partial f}{\partial p_{x}}=\sum_{\vec{p}^{\prime}, s^{\prime}} S\left(\vec{p}, s \leftrightarrow \vec{p}^{\prime}, s^{\prime}\right)\left(f-f^{\prime}\right),
$$

where we assume elastic scattering so that the scattering rates are the same in both directions, i.e.,

$$
S\left(\vec{p}, s \rightarrow \vec{p}^{\prime}, s^{\prime}\right)=S\left(\vec{p}^{\prime}, s^{\prime} \rightarrow \vec{p}, s\right) \equiv S\left(\vec{p}, s \leftrightarrow \vec{p}^{\prime}, s^{\prime}\right) .
$$

Here, $f \equiv f(x, t, \vec{p}, s)$ is the occupation factor of a state for a particular position $x$, time $t$, momentum $\vec{p}$, and spin index $s= \pm 1 ; f^{\prime} \equiv f\left(x, t, \vec{p}^{\prime}, s^{\prime}\right)$ with momentum $\vec{p}^{\prime}$ and spin index $s^{\prime}= \pm 1 ; v_{x}=\partial E / \partial p_{x}$ is the $x$ component of the group velocity; $F_{x}=-\partial E / \partial x$ is the force on electrons along the $\hat{x}$ direction; and $E$ is the total energy. Note that the spin index +1 and -1 corresponds to up $(U)$ and down (D) spin polarized states for a particular $\vec{p}$.

\section{B. Occupation factor}

We write the occupation factor $f$ in terms of an electrochemical potential $\mu \equiv \mu(x, t, \vec{p}, s)$, in the form

$$
f(x, t, \vec{p}, s)=\frac{1}{1+\exp \left\{[E(x, t, \vec{p}, s)-\mu(x, t, \vec{p}, s)] / k_{B} T\right\}},
$$

where $k_{B}$ is the Boltzmann constant and $T$ is the temperature. 


\section{Linearization}

We apply a variable transform $\xi=E-\mu$ on the lefthand side of Eq. (31). On the right-hand side of Eq. (31), we expand both $f$ and $f^{\prime}$ into Taylor series around

$$
f_{0}=\frac{1}{1+\exp \left\{\left[E(x, \vec{p}, s)-\mu_{0}\right] / k_{B} T\right\}},
$$

with constant electrochemical potential $\mu_{0}$, and we apply linear response approximation. Thus, Eq. (31) can be written as (see Appendix E for details of the derivation)

$$
\begin{aligned}
& \left(-\frac{\partial f_{0}}{\partial E}\right)\left(\frac{\partial \mu}{\partial t}-\frac{\partial E}{\partial t}+v_{x} \frac{\partial \mu}{\partial x}+F_{x} \frac{\partial \mu}{\partial p_{x}}\right) \\
& =-\sum_{\overrightarrow{p^{\prime}, s^{\prime}}} S\left(\vec{p}, s \leftrightarrow \vec{p}^{\prime}, s^{\prime}\right)\left(-\frac{\partial f_{0}}{\partial E}\right)\left(\mu-\mu^{\prime}\right) .
\end{aligned}
$$

We assume that there are no internal fields in the present discussion. Hence, $F_{x}$ comes from the applied voltage and the term $F_{x}\left(\partial \mu / \partial p_{x}\right)$ depends on the higher order of the applied voltage, which can be neglected in the linear response regime [64]. Thus, Eq. (34) is given by

$$
\begin{aligned}
& \left(-\frac{\partial f_{0}}{\partial E}\right)\left(\frac{\partial \mu}{\partial t}-\frac{\partial E}{\partial t}+v_{x} \frac{\partial \mu}{\partial x}\right) \\
& =-\sum_{\vec{p}^{\prime}, s^{\prime}} S\left(\vec{p}, s \leftrightarrow \vec{p}^{\prime}, s^{\prime}\right)\left(-\frac{\partial f_{0}}{\partial E}\right)\left(\mu-\mu^{\prime}\right) .
\end{aligned}
$$

The term $\partial E / \partial t$ can be evaluated from the dispersion relation of a given Hamiltonian in the semiclassical approximation as discussed below.

\section{Dispersion relation}

We start from the following Rashba Hamiltonian:

$$
\mathcal{H}=\frac{|\vec{p}-q \vec{A}|^{2}}{2 m} I_{2 \times 2}-v_{0}[\vec{\sigma} \times(\vec{p}-q \vec{A})] \cdot \hat{y}+U_{E} I_{2 \times 2}
$$

Here, $I_{2 \times 2}$ is a $2 \times 2$ identity matrix; $\vec{p}$ and $\vec{A}$ are the momentum and vector magnetic potential, respectively, in the $z-x$ plane; $\vec{\sigma}$ is the Pauli's matrices; $v_{0}$ is the Rashba coefficient; $U_{E}$ is the electrostatic potential; $m$ is the electron mass; and $q$ is the electron charge.

Eigenstates of Eq. (36) are given by

$$
E(\vec{p}, s)=\frac{|\vec{p}-q \vec{A}|^{2}}{2 m}-s v_{0}|\vec{p}-q \vec{A}|+U_{E},
$$

where $\vec{p}$ is confined to the $z-x$ plane.
We assume that $U_{E}$ and $\vec{A}$ vary slowly with $x$ and $t$, so that in the semiclassical approximation, we have

$$
\begin{aligned}
E(x, t, \vec{p}, s)= & \frac{|\vec{p}-q \vec{A}(x, t)|^{2}}{2 m} \\
& -s v_{0}|\vec{p}-q \vec{A}(x, t)|+U_{E}(x, t) .
\end{aligned}
$$

Differentiating Eq. (38) with respect to $t$ yields

$$
\frac{\partial E}{\partial t}=\vec{v} \cdot\left(-q \frac{\partial \vec{A}}{\partial t}\right)+\frac{\partial U_{E}}{\partial t},
$$

where $\vec{v}=\nabla_{\vec{p}} E$ (see Appendix $\mathrm{F}$ for the derivation).

The electrostatic potential $U_{E}$ and the vector magnetic potential $\vec{A}$ on the structure of interest can be evaluated from the theory of electromagnetism.

\section{E. From potentials to charge and current}

The electrostatic potential $U_{E}$ is related to the total charge $Q$ in the channel by the electrostatic capacitance $C_{E}$ of the structure under consideration, given by

$$
\frac{U_{E}}{q}=\frac{Q}{C_{E}} .
$$

We assume that the charge current $I_{c}$ flows along the $\hat{x}$ direction, which is uniform in the channel. The vector magnetic potential $\vec{A}$ is related to $I_{c}$ by the magnetic inductance $L_{M}$ of the channel, given by

$$
\vec{A} \equiv \hat{x} A_{x}=\hat{x} L_{M} I_{c} .
$$

Thus, Eq. (39) can be written as

$$
\frac{\partial E}{\partial t}=-q v_{x} L_{M} \frac{\partial I_{c}}{\partial t}+\frac{q}{C_{E}} \frac{\partial Q}{\partial t} .
$$

We combine Eq. (35) with Eq. (42) to get

$$
\begin{gathered}
\left(-\frac{\partial f_{0}}{\partial E}\right)\left(\frac{\partial \mu}{\partial t}+v_{x} \frac{\partial \mu}{\partial x}+q v_{x} L_{M} \frac{\partial I_{c}}{\partial t}-\frac{q}{C_{E}} \frac{\partial Q}{\partial t}\right) \\
=-\sum_{\overrightarrow{p^{\prime}, s^{\prime}}} S\left(\vec{p}, s \leftrightarrow \vec{p}^{\prime}, s^{\prime}\right)\left(-\frac{\partial f_{0}}{\partial E}\right)\left(\mu-\mu^{\prime}\right) .
\end{gathered}
$$

\section{F. Classification}

We classify all $\vec{p}, s$ states into four groups based on the sign of $v_{x}(+$ or - ) and the spin index $s= \pm 1$, given by

$$
\Re:\left\{\begin{array}{l}
U^{+} \in\left\{\vec{p}, s \mid v_{x}>0, s=+1\right\}, \\
D^{-} \in\left\{\vec{p}, s \mid v_{x}<0, s=-1\right\}, \\
U^{-} \in\left\{\vec{p}, s \mid v_{x}<0, s=+1\right\}, \quad \text { and } \\
D^{+} \in\left\{\vec{p}, s \mid v_{x}>0, s=-1\right\},
\end{array}\right.
$$

where $s=+1$ and -1 denote up $(U)$ and down $(D)$ spins with respect to the spin quantization axis defined by $\hat{y} \times$ 
$(\vec{p}-q \vec{A})$, which is different for each direction of $\vec{p}$. Such classification can be mapped onto the two Fermi circles of a Rashba channel [see Fig. 5(a)]. The large circle corresponds to $U^{+}$and $D^{-}$groups that share the same number of modes $n_{m}\left(U^{+}\right)=n_{m}\left(D^{-}\right)=M$, satisfying the timereversal symmetry. Similarly, the small circle corresponds to $U^{-}$and $D^{+}$groups sharing the same number of modes $n_{m}\left(U^{-}\right)=n_{m}\left(D^{+}\right)=N$. Note that the eigenstates belonging to each of the four half Fermi circles in Fig. 5(a) have an average spin polarization along the $\hat{z}$ direction with an averaging factor of $\alpha=2 / \pi$, which we will use later when writing spin currents and voltages.

\section{G. Averaging}

We define the thermal average of a variable $\psi \equiv \psi(\vec{p}, s)$ within each of the groups $\vec{p}, s \in \Re$ as

$$
\langle\psi\rangle_{\vec{p}, s \in \Re}=\frac{\sum_{\vec{p}, s \in \Re}\left(-\frac{\partial f_{0}}{\partial E}\right) \psi(\vec{p}, s)}{\sum_{\vec{p}, s \in \Re}\left(-\frac{\partial f_{0}}{\partial E}\right)} .
$$

We sum both sides of Eq. (43) over all $\vec{p}, s$ states within the range $\vec{p}, s \in \mathfrak{R}$ in the $z$ - $x$ plane as

$$
\begin{aligned}
& \frac{D_{0}(\Re)}{2} \frac{\partial\langle\mu\rangle}{\partial t}+\frac{D_{0}(\Re)}{2} \frac{\partial\left\langle v_{x} \mu\right\rangle}{\partial x} \\
& \quad+\frac{q D_{0}(\Re)}{2}\left\langle v_{x}\right\rangle L_{M} \frac{\partial I_{c}}{\partial t}-\frac{q}{C_{E}} \frac{D_{0}(\Re)}{2} \frac{\partial Q}{\partial t} \\
& \quad=-\sum_{\vec{p}, s \in \Re} \sum_{\vec{p}^{\prime}, s^{\prime}} S\left(\vec{p}, s \leftrightarrow \vec{p}^{\prime}, s^{\prime}\right)\left(-\frac{\partial f_{0}}{\partial E}\right)\left(\mu-\mu^{\prime}\right) .
\end{aligned}
$$

Here, $D_{0}(\Re)$ is the thermally averaged density of states within $\vec{p}, s \in \Re$, given by

$$
\frac{D_{0}(\Re)}{2}=\sum_{\vec{p}, s \in \Re}\left(-\frac{\partial f_{0}}{\partial E}\right),
$$

where the factor of 2 appeared since we are summing over all $s$ states. Note that the time-reversal symmetry requires that

$$
D_{0}\left(U^{+}\right)=D_{0}\left(D^{-}\right) \text {and } D_{0}\left(U^{-}\right)=D_{0}\left(D^{+}\right) .
$$

We make the following assumption in Eq. (46):

$$
\left\langle v_{x} \mu\right\rangle \approx\left\langle v_{x}\right\rangle\langle\mu\rangle
$$
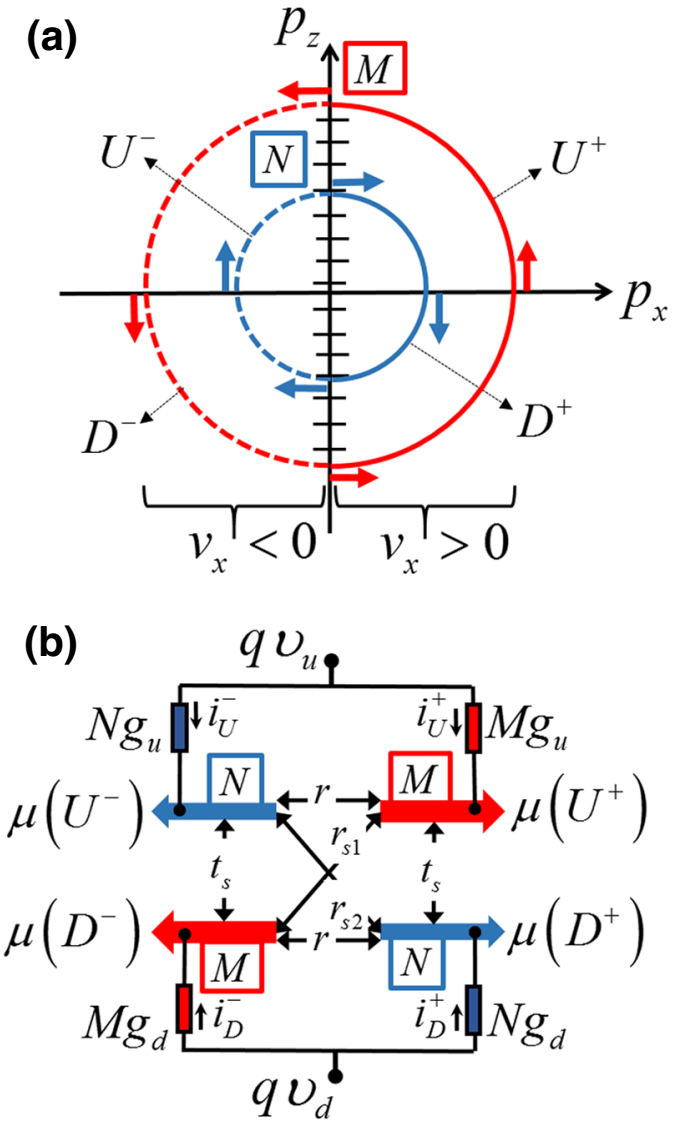

FIG. 5. (a) Two Fermi circles of a Rashba channel with spinmomentum locking having opposite spin polarizations at a given energy $E_{F}$. The left (right) half of each circle represents negative (positive) group velocity. The large circle corresponds to the large number of modes $M$ in the channel and has a net spin polarization along $+\hat{z}(-\hat{z})$ on right (left) half. The small circle corresponds to a smaller number of modes $N$ and has net spin polarization along $-\hat{z}(+\hat{z})$ on the right (left) half. We classify all electronic states into four groups based on the spin index [up $(U)$, down $(D)$ ] and the sign of the $x$ component of the group velocity $(+,-)$. (b) We assign four average electrochemical potentials for these four groups: $\mu\left(U^{+}\right), \mu\left(U^{-}\right), \mu\left(D^{+}\right)$, and $\mu\left(D^{-}\right)$. External contact is modeled as up $\left(v_{u}\right)$ and down $\left(v_{d}\right)$ spin voltages applied to up and down states of the channel through up $\left(g_{u}\right)$ and down $\left(g_{d}\right)$ spin conductances per mode per unit length, respectively. Four different currents $\left(i_{U}^{+}, i_{D}^{+}, i_{U}^{-}\right.$, and $\left.i_{D}^{-}\right)$enter the four different groups in the channel. The contact can be either normal metal $g_{u}=g_{d}$ or ferromagnet $g_{u} \neq g_{d}$.

which yields

$$
\begin{aligned}
& \frac{D_{0}(\Re)}{2} \frac{\partial\langle\mu\rangle}{\partial t}+\frac{D_{0}(\Re)}{2}\left\langle v_{x}\right\rangle \frac{\partial\langle\mu\rangle}{\partial x}+\frac{q D_{0}(\Re)}{2}\left\langle v_{x}\right\rangle L_{M} \frac{\partial I_{c}}{\partial t} \\
& -\frac{q}{C_{E}} \frac{D_{0}(\Re)}{2} \frac{\partial Q}{\partial t} \\
& \quad=-\sum_{\vec{p}, s \in \Re} \sum_{\vec{p}^{\prime}, s^{\prime}} S\left(\vec{p}, s \leftrightarrow \vec{p}^{\prime}, s^{\prime}\right)\left(-\frac{\partial f_{0}}{\partial E}\right)\left(\mu-\mu^{\prime}\right)+\frac{i_{\mathrm{ext}}}{q} .
\end{aligned}
$$


Note that the term $i_{\mathrm{ext}} / q$ on the right-hand side has been added to take into account the total current entering into the $\vec{p}, s \in \Re$ states from an external contact.

The number of modes within $\vec{p}, s \in \Re$ in the channel is given by [64]

$$
n_{m}(\Re)=\frac{h D_{0}\left|\left\langle v_{x}\right\rangle\right|}{2 L},
$$

where $\left|\left\langle v_{x}\right\rangle\right|$ is the magnitude of $\left\langle v_{x}\right\rangle$ and $L$ is the channel length. Note that $\left|\left\langle v_{x}\right\rangle\right|$ is the same in all four groups for the Rashba Hamiltonian considered here (see Appendix F). Thus, Eq. (49) can be written as

$$
\begin{aligned}
& \frac{n_{m}(\Re)}{\left|\left\langle v_{x}\right\rangle\right|} \frac{\partial\langle\mu\rangle}{\partial t}+\operatorname{sgn}\left(\left\langle v_{x}\right\rangle\right) n_{m}(\Re) \frac{\partial\langle\mu\rangle}{\partial x}-\frac{q}{C_{E}} \frac{n_{m}(\Re)}{\left|\left\langle v_{x}\right\rangle\right|} \frac{\partial Q}{\partial t} \\
& \quad+\operatorname{sgn}\left(\left\langle v_{x}\right\rangle\right) q n_{m}(\Re) L_{M} \frac{\partial I_{c}}{\partial t}=\tilde{S}(\Re)+\frac{h}{q} \frac{i_{\mathrm{ext}}}{L}
\end{aligned}
$$

where

$$
\tilde{S}(\Re)=-\frac{h}{L} \sum_{\vec{p}, s \in \Re} \sum_{\vec{p}^{\prime}, s^{\prime}} S\left(\vec{p}, s \leftrightarrow \vec{p}^{\prime}, s^{\prime}\right)\left(-\frac{\partial f_{0}}{\partial E}\right)\left(\mu-\mu^{\prime}\right) .
$$

Equation (51) applies to each group in Eq. (44) with an average electrochemical potential given by $\langle\mu\rangle_{\vec{p}, s \in U^{+}} \equiv$ $\mu\left(U^{+}\right),\langle\mu\rangle_{\vec{p}, s \in D^{-}} \equiv \mu\left(D^{-}\right),\langle\mu\rangle_{\vec{p}, s \in U^{-}} \equiv \mu\left(U^{-}\right)$, and $\langle\mu\rangle_{\vec{p}, s \in D^{+}} \equiv \mu\left(D^{+}\right)$, respectively.

\section{H. Scattering matrix}

We make the following assumption:

$$
\langle S \mu\rangle \approx\langle S\rangle\langle\mu\rangle,
$$

which in conjunction with Eq. (52) becomes

$$
\begin{aligned}
\tilde{S}(\Re)= & \hat{S}_{\Re \leftrightarrow U^{+}}\left[\left\langle\mu\left(U^{+}\right)\right\rangle-\langle\mu(\Re)\rangle\right] \\
& +\hat{S}_{\Re \leftrightarrow D^{-}}\left[\left\langle\mu\left(D^{-}\right)\right\rangle-\langle\mu(\Re)\rangle\right] \\
& +\hat{S}_{\Re \leftrightarrow U^{-}}\left[\left\langle\mu\left(U^{-}\right)\right\rangle-\langle\mu(\Re)\rangle\right] \\
& +\hat{S}_{\Re \leftrightarrow D^{+}}\left[\left\langle\mu\left(D^{+}\right)\right\rangle-\langle\mu(\Re)\rangle\right],
\end{aligned}
$$

with

$$
\hat{S}_{\Re \leftrightarrow \Re^{\prime}}=\frac{h}{L} \sum_{\vec{p}, s \in \Re} \sum_{\vec{p}^{\prime}, s^{\prime} \in \Re^{\prime}}\left(-\frac{\partial f_{0}}{\partial E}\right) S\left(\vec{p}, s \leftrightarrow \vec{p}^{\prime}, s^{\prime}\right) .
$$

We can evaluate Eq. (54) for each of the groups in Eq. (44), i.e., $\Re \equiv U^{+}, D^{-}, U^{-}$, and $D^{+}$, which together in the $\left\{\mu\left(U^{+}\right), \mu\left(D^{-}\right), \mu\left(U^{-}\right), \mu\left(D^{+}\right)\right\}^{T}$ basis become the following matrix (see Appendix $\mathrm{G}$ for details):

$$
[S]=\left[\begin{array}{cccc}
-u_{1} & \hat{S}_{U^{+} \leftrightarrow D^{-}} & \hat{S}_{U^{+} \leftrightarrow U^{-}} & \hat{S}_{U^{+} \leftrightarrow D^{+}} \\
\hat{S}_{U^{+} \leftrightarrow D^{-}} & -u_{1}^{\prime} & \hat{S}_{D^{-} \leftrightarrow U^{-}} & \hat{S}_{D^{-} \leftrightarrow D^{+}} \\
\hat{S}_{U^{+} \leftrightarrow U^{-}} & \hat{S}_{D^{-} \leftrightarrow U^{-}} & -u_{2} & \hat{S}_{U^{-} \leftrightarrow D^{+}} \\
\hat{S}_{U^{+} \leftrightarrow D^{+}} & \hat{S}_{D^{-} \leftrightarrow D^{+}} & \hat{S}_{U^{-} \leftrightarrow D^{+}} & -u_{2}^{\prime}
\end{array}\right],
$$

where

$$
\begin{aligned}
u_{1} & =\hat{S}_{U^{+} \leftrightarrow D^{-}}+\hat{S}_{U^{+} \leftrightarrow U^{-}}+\hat{S}_{U^{+} \leftrightarrow D^{+}}, \\
u_{1}^{\prime} & =\hat{S}_{U^{+} \leftrightarrow D^{-}}+\hat{S}_{D^{-} \leftrightarrow U^{-}}+\hat{S}_{D^{-} \leftrightarrow D^{+}}, \\
u_{2} & =\hat{S}_{U^{+} \leftrightarrow U^{-}}+\hat{S}_{D^{-} \leftrightarrow U^{-}}+\hat{S}_{U^{-} \leftrightarrow D^{+}}, \\
\text {and } u_{2}^{\prime} & =\hat{S}_{U^{+} \leftrightarrow D^{+}}+\hat{S}_{D^{-} \leftrightarrow D^{+}}+\hat{S}_{U^{-} \leftrightarrow D^{+}} .
\end{aligned}
$$

The scattering matrix is such that the sum of each column is zero, satisfying the charge conservation, and the sum of each row is zero, satisfying the zero current requirement under equal potential.

In addition, the time-reversal symmetry requires that

$$
\hat{S}_{U^{+} \leftrightarrow U^{-}}=\hat{S}_{D^{-} \leftrightarrow D^{+}} \quad \text { and } \quad \hat{S}_{U^{+} \leftrightarrow D^{+}}=\hat{S}_{D^{-} \leftrightarrow U^{-}} .
$$

There are three types of scattering processes considered in the channel: (a) reflection with spin flip $r_{s 1}=$ $\hat{S}_{U^{+} \leftrightarrow D^{-}}$and $r_{s 2}=\hat{S}_{U^{-} \leftrightarrow D^{+}}$, (b) reflection without spin flip $r=\hat{S}_{U^{+} \leftrightarrow U^{-}}=\hat{S}_{D^{-} \leftrightarrow D^{+}}$, and (c) transmission with spin flip $t_{s}=\hat{S}_{U^{+} \leftrightarrow D^{+}}=\hat{S}_{D^{-} \leftrightarrow U^{-}}$. They are given by

$$
r_{s 1}=\frac{h}{L} \sum_{\vec{p}, s \in U^{+}} \sum_{\vec{p}^{\prime}, s^{\prime} \in D^{-}}\left(-\frac{\partial f_{0}}{\partial E}\right) S\left(\vec{p}, s \leftrightarrow \vec{p}^{\prime}, s^{\prime}\right),
$$

$$
r_{s 2}=\frac{h}{L} \sum_{\vec{p}, s \in U^{-}} \sum_{\vec{p}^{\prime}, s^{\prime} \in D^{+}}\left(-\frac{\partial f_{0}}{\partial E}\right) S\left(\vec{p}, s \leftrightarrow \vec{p}^{\prime}, s^{\prime}\right),
$$

$$
\begin{aligned}
r & =\frac{h}{L} \sum_{\vec{p}, s \in U^{+}} \sum_{\vec{p}^{\prime}, s^{\prime} \in U^{-}}\left(-\frac{\partial f_{0}}{\partial E}\right) S\left(\vec{p}, s \leftrightarrow \vec{p}^{\prime}, s^{\prime}\right), \\
& =\frac{h}{L} \sum_{\vec{p}, s \in D^{-}} \sum_{\vec{p}^{\prime}, s^{\prime} \in D^{+}}\left(-\frac{\partial f_{0}}{\partial E}\right) S\left(\vec{p}, s \leftrightarrow \vec{p}^{\prime}, s^{\prime}\right),
\end{aligned}
$$

and

$$
\begin{aligned}
t_{s} & =\frac{h}{L} \sum_{\vec{p}, s \in U^{+}} \sum_{\vec{p}^{\prime}, s^{\prime} \in D^{+}}\left(-\frac{\partial f_{0}}{\partial E}\right) S\left(\vec{p}, s \leftrightarrow \vec{p}^{\prime}, s^{\prime}\right), \\
& =\frac{h}{L} \sum_{\vec{p}, s \in D^{-}} \sum_{\vec{p}^{\prime}, s^{\prime} \in U^{-}}\left(-\frac{\partial f_{0}}{\partial E}\right) S\left(\vec{p}, s \leftrightarrow \vec{p}^{\prime}, s^{\prime}\right) .
\end{aligned}
$$


Equation (51) for each group in Eq. (44) is given as

$$
\begin{gathered}
\frac{1}{\left|\left\langle v_{x}\right\rangle\right|} \frac{\partial}{\partial t}\left\{\begin{array}{l}
M \tilde{\mu}\left(U^{+}\right) \\
M \tilde{\mu}\left(D^{-}\right) \\
N \tilde{\mu}\left(U^{-}\right) \\
N \tilde{\mu}\left(D^{+}\right)
\end{array}\right\}+\frac{\partial}{\partial x}\left\{\begin{array}{c}
M \tilde{\mu}\left(U^{+}\right) \\
-M \tilde{\mu}\left(D^{-}\right) \\
-N \tilde{\mu}\left(U^{-}\right) \\
N \tilde{\mu}\left(D^{+}\right)
\end{array}\right\} \\
=\left[\begin{array}{cccc}
-u_{1} & r_{s 1} & r & t_{s} \\
r_{s 1} & -u_{1} & t_{s} & r \\
r & t_{s} & -u_{2} & r_{s 2} \\
t_{s} & r & r_{s 2} & -u_{2}
\end{array}\right]\left\{\begin{array}{l}
\tilde{\mu}\left(U^{+}\right) \\
\tilde{\mu}\left(D^{-}\right) \\
\tilde{\mu}\left(U^{-}\right) \\
\tilde{\mu}\left(D^{+}\right)
\end{array}\right\} \\
-q L_{M} \frac{\partial I_{c}}{\partial t}\left\{\begin{array}{c}
M \\
-M \\
-N \\
N
\end{array}\right\}+\frac{q}{\left|\left\langle v_{x}\right\rangle\right| C_{E}} \frac{\partial Q}{\partial t}\left\{\begin{array}{l}
M \\
M \\
N \\
N
\end{array}\right\} \\
+\frac{h}{q}\left\{\begin{array}{c}
i_{U}^{+} \\
i_{D}^{-} \\
i_{U}^{-} \\
i_{D}^{+}
\end{array}\right\} .
\end{gathered}
$$

Note that the electrochemical potentials are referenced with respect to the constant $\mu_{0}$, i.e., $\tilde{\mu}=\mu-\mu_{0} . i_{U}^{+}, i_{D}^{-}$, $i_{U}^{-}$, and $i_{D}^{+}$are the currents per unit length entering into the four groups from an external contact [see Fig. 5(b)], which are given as [52]

$$
\begin{aligned}
i_{U}^{+} & =\frac{q^{2}}{h} M g_{u}\left[v_{u}-\frac{\tilde{\mu}\left(U^{+}\right)}{q}\right], \\
i_{D}^{+} & =\frac{q^{2}}{h} N g_{d}\left[v_{d}-\frac{\tilde{\mu}\left(D^{+}\right)}{q}\right], \\
i_{U}^{-} & =\frac{q^{2}}{h} N g_{u}\left[v_{u}-\frac{\tilde{\mu}\left(U^{-}\right)}{q}\right], \\
\text { and } \quad i_{D}^{-} & =\frac{q^{2}}{h} M g_{d}\left[v_{d}-\frac{\tilde{\mu}\left(D^{-}\right)}{q}\right] .
\end{aligned}
$$

Here, $v_{u}$ and $v_{d}$ are the up and down spin voltages at the external contact, respectively. $g_{u}$ and $g_{d}$ are the up and down spin conductances per unit mode per unit length. The contact can be either NM $\left(g_{u}=g_{d}\right)$ or FM $\left(g_{u} \neq g_{d}\right)$. In steady state, Eq. (59) reduces to our prior model in Ref. [52].

\section{Conversion to charge-spin basis}

The charge and spin voltages and currents in the channel are defined in terms of the four average electrochemical potentials as

$$
\left\{\begin{array}{l}
I_{c} R_{B} \\
2 V_{s} \\
I_{S} R_{B} \\
2 V_{c}
\end{array}\right\}=\frac{q}{h} R_{B}\left[\begin{array}{cccc}
1 & -1 & -1 & 1 \\
\alpha & -\alpha & \alpha & -\alpha \\
\frac{1}{\alpha} & \frac{1}{\alpha} & -\frac{1}{\alpha} & -\frac{1}{\alpha} \\
1 & 1 & 1 & 1
\end{array}\right]\left\{\begin{array}{l}
M \tilde{\mu}\left(U^{+}\right) \\
M \tilde{\mu}\left(D^{-}\right) \\
N \tilde{\mu}\left(U^{-}\right) \\
N \tilde{\mu}\left(D^{+}\right)
\end{array}\right\},
$$

where $R_{B}$ is the ballistic resistance of the channel given in Eq. (5k) and $\alpha$ is an angular averaging factor. See Appendix $\mathrm{H}$ for the derivation of Eq. (61).

The net $z$-spin polarization (or $z$-spin voltage) is expected to be lower due to the angular distribution of the spin polarization of the eigenstates on the half Fermi circles indicated by $U^{+}, U^{-}, D^{+}$, and $D^{-}$in Fig. 5(a) and the details of the scattering mechanisms. In order to take into account this scenario, we multiply the second row of Eq. (61) with a factor $0 \leq \alpha \leq 1$, which appears in Eq. (19), to indicate a lowering of the charge-currentinduced spin voltage in the channel from the ideal value. Onsager reciprocity requires that the spin-current-induced charge voltage in the channel be lowered by the same factor $\alpha$ as shown in Eq. (20), which has been taken into account by multiplying $1 / \alpha$ to the third row of Eq. (61). In the simplest approximation, the angle between the $z$ axis and the spin polarization of the eigenstates of a particular half Fermi circle in Fig. 5(a) varies from $-\pi / 2$ to $+\pi / 2$, which yields $\alpha=2 / \pi$.

Combining Eq. (59) with Eq. (61) yields

$$
\begin{aligned}
& \frac{1}{\left|\left\langle v_{x}\right\rangle\right|} \frac{\partial}{\partial t}\left[\begin{array}{cccc}
0 & 0 & 0 & 1 \\
0 & 0 & \alpha^{2} & 0 \\
0 & \frac{1}{\alpha^{2}} & 0 & 0 \\
1 & 0 & 0 & 0
\end{array}\right]\left\{\begin{array}{l}
I_{c} R_{B} \\
2 V_{s} \\
I_{s} R_{B} \\
2 V_{c}
\end{array}\right\}+\frac{\partial}{\partial x}\left\{\begin{array}{l}
I_{c} R_{B} \\
2 V_{s} \\
I_{s} R_{B} \\
2 V_{c}
\end{array}\right\} \\
& =\left[\begin{array}{cccc}
0 & 0 & 0 & 0 \\
0 & 0 & -\frac{2 \alpha^{2}}{\lambda_{0}} & \frac{2 \alpha p_{0}}{\lambda_{0}} \\
\frac{2}{\alpha \lambda_{s}^{\prime}} & -\frac{2}{\alpha^{2} \lambda_{s}} & 0 & 0 \\
-\frac{2}{\lambda} & \frac{2}{\alpha \lambda^{\prime}} & 0 & 0
\end{array}\right]\left\{\begin{array}{l}
I_{c} R_{B} \\
2 V_{s} \\
I_{s} R_{B} \\
2 V_{c}
\end{array}\right\} \\
& -2 L_{M} \frac{\partial I_{c}}{\partial t}\left\{\begin{array}{l}
0 \\
0 \\
\frac{p_{0}}{\alpha} \\
1
\end{array}\right\}+\frac{1}{\left|\left\langle v_{x}\right\rangle\right|} \frac{2}{C_{E}} \frac{\partial Q}{\partial t}\left\{\begin{array}{l}
1 \\
\alpha p_{0} \\
0 \\
0
\end{array}\right\} \\
& +\left\{\begin{array}{l}
R_{B} i^{c} \\
2 \Delta v^{s} \\
R_{B} i^{s} \\
2 \Delta v^{c}
\end{array}\right\},
\end{aligned}
$$


with the external contact terms given by

$$
\begin{aligned}
& i^{c}=i_{U}^{+}+i_{D}^{-}+i_{U}^{-}+i_{D}^{+}, \\
& i^{s}=\frac{1}{\alpha}\left(i_{U}^{+}-i_{D}^{-}+i_{U}^{-}-i_{D}^{+}\right), \\
& \Delta v^{c}=\frac{R_{B}}{2}\left(i_{U}^{+}-i_{D}^{-}-i_{U}^{-}+i_{D}^{+}\right), \quad \text { and } \\
& \Delta v^{s}=\frac{\alpha R_{B}}{2}\left(i_{U}^{+}+i_{D}^{-}-i_{U}^{-}-i_{D}^{+}\right) .
\end{aligned}
$$

Equation (63) combined with Eq. (60) yields Eqs. (8) and (9).

\section{J. Continuity equation}

The term $\partial Q / \partial t$ on the right-hand side of Eq. (62) is related to the charge currents according to the continuity equation given by

$$
\frac{\partial Q}{\partial t}+\frac{\partial I_{c}}{\partial x}=i^{c} .
$$

The first row of Eq. (62) combined with Eq. (64) becomes

$$
\frac{\partial}{\partial t} V_{c}=\left(\frac{\left|\left\langle v_{x}\right\rangle\right| R_{B}}{2}+\frac{1}{C_{E}}\right)\left(i^{c}-\frac{\partial}{\partial x} I_{c}\right),
$$

which is the first equation in Eq. (6).

The second row of Eq. (62) combined with Eq. (64) becomes

$$
\begin{aligned}
\alpha^{2} \frac{R_{B}}{2\left|\left\langle v_{x}\right\rangle\right|} \frac{\partial}{\partial t} I_{s}+\frac{\partial}{\partial x} V_{s} \\
=-\frac{\alpha^{2} R_{B}}{\lambda_{0}} I_{s}+\frac{2 \alpha p_{0}}{\lambda_{0}} V_{c} \\
\quad+\alpha p_{0} \frac{1}{\left|\left\langle v_{x}\right\rangle\right|} \frac{1}{C_{E}}\left(i^{c}-\frac{\partial I_{c}}{\partial x}\right)+\Delta v^{s} .
\end{aligned}
$$

We replace the expression for $i^{c}-(\partial / \partial x) I_{c}$ from Eq. (65) to get the second equation in Eq. (7). For contact conductance $G_{0} \rightarrow 0$, Eqs. (6) and (7) reduce to Eqs. (2) and (3), respectively.

\section{K. Mean free paths}

We have three distinct mean free paths in Eq. (62), given by

$$
\begin{aligned}
& \frac{1}{\lambda}=\frac{1}{2}\left(\frac{r_{s 2}}{N}+\frac{r_{s 1}}{M}\right)+\frac{r}{2}\left(\frac{1}{N}+\frac{1}{M}\right), \\
& \frac{1}{\lambda_{0}}=\frac{r+t_{s}}{2}\left(\frac{1}{N}+\frac{1}{M}\right), \quad \text { and } \\
& \frac{1}{\lambda_{s}}=\frac{1}{2}\left(\frac{r_{s 2}}{N}+\frac{r_{s 1}}{M}\right)+\frac{t_{s}}{2}\left(\frac{1}{N}+\frac{1}{M}\right),
\end{aligned}
$$

where $\lambda, \lambda_{0}$, and $\lambda_{s}$ determine the series charge resistance $R_{c}$ [see Eq. (5c)], the series spin resistance $R_{S}$ [see Eq. (5d)], and the shunt spin conductance $G_{\text {sh }}$ [see (5e)], respectively. Note that $\lambda_{s}$ depends on the spin-flip processes in the channel and determines the shunt conductance $G_{\text {sh }}$ that takes into account the spin relaxation process.

\section{Charge-spin coupling coefficients}

The other terms of Eq. (62) are given by

$$
\begin{aligned}
& \frac{1}{\lambda^{\prime}}=\frac{1}{2}\left(\frac{r_{s 2}}{N}-\frac{r_{s 1}}{M}\right)+\frac{r}{2}\left(\frac{1}{N}-\frac{1}{M}\right) \quad \text { and } \\
& \frac{1}{\lambda_{s}^{\prime}}=\frac{1}{2}\left(\frac{r_{s 2}}{N}-\frac{r_{s 1}}{M}\right)+\frac{t_{s}}{2}\left(\frac{1}{N}-\frac{1}{M}\right),
\end{aligned}
$$

representing coupling coefficients between charge and spin. $\lambda_{s}^{\prime}$ and $\lambda^{\prime}$ cause a charge-induced spin signal and spin-induced charge signal, respectively. Note that the first terms of Eq. (68) indicate a purely scattering-induced spincharge coupling even if $M=N$ (i.e., $p_{0}=0$ ), since $r_{s 1}$ and $r_{s 2}$ are two independent parameters, and there could be situations where $r_{s 1} \neq r_{s 2}$.

In this paper, we restrict ourselves to SML caused by difference between $M$ and $N$ (i.e., $p_{0} \neq 0$ ). We can eliminate the first terms in Eq. (68) by assuming either of the following:

$$
\begin{aligned}
& r_{s 1}=r_{s 2}=r_{s}, \\
& \text { or } \frac{r_{s 1}}{M}=\frac{r_{s 2}}{N} .
\end{aligned}
$$

The first assumption Eq. (69a) when applied to Eq. (68) yields

$$
\begin{aligned}
& \frac{1}{\lambda^{\prime}}=\left(\frac{r_{s}+r}{2}\right)\left(\frac{1}{N}-\frac{1}{M}\right)=\frac{p_{0}}{\lambda} \text { and } \\
& \frac{1}{\lambda_{s}^{\prime}}=\left(\frac{r_{s}+t_{s}}{2}\right)\left(\frac{1}{N}-\frac{1}{M}\right)=\frac{p_{0}}{\lambda_{s}},
\end{aligned}
$$

which, in turn, gives $\lambda_{r}=\lambda$ and $\lambda_{t}=\lambda_{s}$ in Eqs. (5i) and (5j), respectively.

The second assumption Eq. (69b) when applied in Eq. (68) yields

$$
\begin{aligned}
& \frac{1}{\lambda^{\prime}}=\frac{r}{2}\left(\frac{1}{N}-\frac{1}{M}\right)=\frac{p_{0}}{\lambda_{r}} \quad \text { and } \\
& \frac{1}{\lambda_{s}^{\prime}}=\frac{t_{s}}{2}\left(\frac{1}{N}-\frac{1}{M}\right)=\frac{p_{0}}{\lambda_{t}}
\end{aligned}
$$

\section{Comments on the assumptions}

The assumptions in Eqs. (48), (53), and (69) result in an effective change in the transmission-line model parameters in Eqs. (5), but they do not change the models in 
Eqs. (2) and (3) (Fig. 1) and Eqs. (6) and (7) (Fig. 2) themselves. The assumptions made to derive the model can be revisited as the field evolves. However, several predictions from our model for steady state transport [52] have already received support from experiments [16,17,26], suggesting that the assumptions are within the reasonable limits.

\section{SUMMARY}

We propose a two-component (charge and $z$ component of spin) transmission-line model for channels with spin-momentum locking, which is a new addition to our SPICE compatible multiphysics model library [37-39]. The model enables easy analysis of complex geometries involving materials with spin-orbit coupling observed in diverse classes of materials, e.g., topological insulators, Kondo insulators, transition metals, semimetals, oxide interfaces, and narrow band-gap semiconductors. The model is derived from a four-component diffusion equation obtained from the Boltzmann transport equation assuming linear response and elastic scattering in the channel. The four-component diffusion equation uses four average electrochemical potentials based on a classification depending on the sign of the $z$ component of spin [up (U) or down $(D)]$ and the sign of the $x$ component of group velocity $(+$ or -$)$. Such classification can be viewed as an extension of the Valet-Fert equation [51], which uses two electrochemical potentials for $U$ and $D$ states. For a normal metal channel, the time-dependent model presented here decouples into (i) the well-known transmission-line model for charge transport in quantum wires $[50,62,63]$ and (ii) a time-dependent version of the Valet-Fert equation [51] for spin transport. We first derive several results on chargespin interconversion, starting from our model in steady state. The steady-state results show good agreement with existing experiments on diverse materials. We then study the phenomenon of spin-charge separation using our full time-dependent model, especially in the materials with SOC exhibiting SML. Our model shows the expected spincharge separation with two distinct velocities for charge and spin, which persist even in channels exhibiting SML. However, we show that the lower velocity signal is purely spin, while the higher velocity signal is largely charge with an additional spin component proportional to the degree of SML.

\section{ACKNOWLEDGMENTS}

This work was in part supported by FAME, one of six centers of STARnet, a Semiconductor Research Corporation (SRC) program sponsored by MARCO and DARPA and in part by ASCENT, one of six centers in JUMP, a SRC program sponsored by DARPA.

\section{APPENDIX A: STEADY-STATE RESULTS}

This appendix provides the details of the derivation of Eqs. (18) and (23).

\section{Derivation of the resistance matrix}

Potentiometric NM contacts: Equations (8) and (9) for $p_{f}=0$ become

$$
\begin{gathered}
i^{c}=G_{0}\left(v_{c}-V_{c}\right), \\
i^{s}=\frac{G_{0}}{\alpha^{2}}\left(v_{s}-V_{s}\right) \\
\Delta v^{c}=\frac{G_{0} R_{B}}{2 \alpha}\left(p_{0} v_{s}-\frac{\alpha I_{c} R_{B}}{2}\right) \\
\text { and } \Delta v^{s}=\frac{\alpha G_{0} R_{B}}{2}\left(p_{0} v_{c}-\frac{\alpha I_{s} R_{B}}{2}\right)
\end{gathered}
$$

We apply Eqs. (A1a) and (A1b) to Eqs. (A1c) and (A1d), respectively, which yields

$$
\begin{aligned}
& \Delta v^{c}=\frac{\alpha p_{0}}{2 G_{B}} i^{s}+p_{0} \frac{G_{0} R_{B}}{2 \alpha} V_{s}-R_{\mathrm{cont}}^{c} I_{c}, \\
& \Delta v^{s}=\frac{\alpha p_{0}}{2 G_{B}} i^{c}+p_{0} \frac{\alpha G_{0} R_{B}}{2} V_{c}-R_{\mathrm{cont}}^{s} I_{s} .
\end{aligned}
$$

We assume that the contact conductance per unit length $G_{0}$ is very low, such that the potentiometric condition in Eq. (17) is satisfied. Thus, we have

$$
\begin{aligned}
& R_{c} \gg R_{\text {cont }}^{c}, \quad R_{s} \gg R_{\text {cont }}^{s}, \\
& \eta_{c} \gg \frac{G_{0} R_{B}}{2 \alpha}, \quad \text { and } \quad \eta_{s} \gg \frac{\alpha G_{0} R_{B}}{2} .
\end{aligned}
$$

Under this condition, we combine Eq. (16) with Eq. (A2) for $i^{c}=0$, which yields

$$
\begin{gathered}
\frac{d}{d x} I_{c}=0 \\
\frac{d}{d x} V_{c}=-R_{c} I_{c}+p_{0} \eta_{c} V_{s}+\frac{\alpha p_{0}}{2 G_{B}} i^{s} \\
\frac{d}{d x} I_{s}=-G_{\mathrm{sh}} V_{s}+p_{0} \gamma_{s} I_{c}+i^{s} \\
\text { and } \frac{d}{d x} V_{s}=-R_{s} I_{s}+p_{0} \eta_{s} V_{c} .
\end{gathered}
$$

Uniform spin voltage: We assume that the spin voltage $V_{s}$ is uniform in the channel region of interest (from $x=0$ 
to $x=L$, where $L$ is the channel length)

$$
\frac{d}{d x} V_{s}=0
$$

Thus, from Eq. (A3d), we can write

$$
I_{s}=\frac{2 p_{0} G_{B}}{\alpha} V_{c},
$$

where we use the definitions in Eqs. (5d) and (5h).

Differentiating both sides of Eq. (A5) with respect to $x$ and combining with Eqs. (A3b) and (A3c) yields

$$
\begin{gathered}
-G_{\mathrm{sh}} V_{s}+p_{0} \gamma_{s} I_{c}+i^{s}=\frac{2 p_{0} G_{B}}{\alpha} \\
\times\left(-R_{c} I_{c}+p_{0} \eta_{c} V_{s}+\frac{\alpha p_{0}}{2 G_{B}} i^{s}\right),
\end{gathered}
$$

which in conjunction with the definitions in Eq. (5) yields

$$
\begin{aligned}
V_{s}= & \frac{\alpha p_{0}}{2 G_{B}} \frac{\left(1 / \lambda_{t}+1 / \lambda\right)}{\left(1 / \lambda_{s}+p_{0}^{2} / \lambda_{r}\right)} I_{c} \\
& +\frac{\alpha^{2}\left(1-p_{0}^{2}\right)}{4 G_{B}} \frac{1}{\left(1 / \lambda_{s}+p_{0}^{2} / \lambda_{r}\right)} i^{s} .
\end{aligned}
$$

From Eq. (A1b), we have

$$
V_{s}=v_{s}-\frac{\alpha^{2} i^{s}}{G_{0}}
$$

which, when combined with Eq. (A6), becomes

$$
\begin{aligned}
v_{s}= & \frac{\alpha p_{0}}{2 G_{B}} \frac{\left(\frac{1}{\lambda_{t}}+\frac{1}{\lambda}\right)}{\left(\frac{1}{\lambda_{s}}+\frac{p_{0}^{2}}{\lambda_{r}}\right)} I_{c} \\
& +\left[\frac{\alpha^{2}\left(1-p_{0}^{2}\right)}{4 G_{B}} \frac{1}{\left(\frac{1}{\lambda_{s}}+\frac{p_{0}^{2}}{\lambda_{r}}\right)}+\frac{\alpha^{2}}{G_{0}}\right] i^{s} .
\end{aligned}
$$

Charge voltage in the channel: Let us assume that the contact 1 is located at $x=0$ and contact 2 is located at $x=L$ as shown in Fig. 3, where $L$ is the channel length between the two contacts. The charge voltages in the channel at $x=0$ and $x=L$ are $V_{c 1}$ and $V_{c 2}$, respectively. According to (A3a), $I_{c}$ is constant along the channel. Since $V_{S}$ and $i^{s}$ are also uniform along the channel, we can integrate
Eq. (A3b) from $x=0$ to $x=L$ as

$$
V_{c 1}-V_{c 2}=\frac{L}{\lambda} R_{B} I_{c}-\frac{2 p_{0} L}{\alpha \lambda_{r}} V_{s}-\frac{\alpha p_{0}}{2 G_{B}} i^{s} L .
$$

From Eq. (A1a), we can write the equation for $V_{c 1}$ in terms of the terminal voltage $v_{c 1}$ of contact 1 at $x=0$ as

$$
V_{c 1}=v_{c 1}-\frac{I_{c}}{G_{0}^{\prime \prime}},
$$

noting that $i^{c}=I_{c}$. Here, $G_{0}^{\prime \prime}$ is the contact conductance of contact 1 . Similarly, we can write an equation for contact 2 , noting that $i^{c}=-I_{c}$, given by

$$
V_{c 2}=v_{c 2}+\frac{I_{c}}{G_{0}^{\prime \prime}}
$$

where we assume that contacts 1 and 2 have the same conductance.

We combine Eq. (A9) with Eqs. (A6), (A10), and (A11) to have

$$
\begin{aligned}
v_{c 1}-v_{c 2}= & \left\{\left[\frac{L}{\lambda}-\frac{p_{0}^{2} L}{\lambda_{r}} \frac{\left(\frac{1}{\lambda_{t}}+\frac{1}{\lambda}\right)}{\left(\frac{1}{\lambda_{s}}+\frac{p_{0}^{2}}{\lambda_{r}}\right)}\right] R_{B}+\frac{2}{G_{0}{ }^{\prime \prime}}\right\} I_{c} \\
& -\frac{\alpha p_{0}}{2 G_{B}} \frac{\left(\frac{1}{\lambda_{r}}+\frac{1}{\lambda_{s}}\right)}{\left(\frac{1}{\lambda_{s}}+\frac{p_{0}^{2}}{\lambda_{r}}\right)} i^{s} L .
\end{aligned}
$$

Scattering condition: We assume that the reflection with a spin-flip scattering mechanism is dominant in the channel

$$
r_{s 1,2} \gg r, t_{s} .
$$

This condition in Eqs. (67), (70), and (68) yields

$$
\frac{1}{\lambda} \approx \frac{1}{\lambda_{s}}, \quad \text { and } \quad \frac{1}{\lambda}, \frac{1}{\lambda_{s}} \gg \frac{1}{\lambda_{0}}, \frac{1}{\lambda_{r}}, \frac{1}{\lambda_{t}} .
$$

Applying Eq. (A14) to Eqs. (A12) and (A8), we have the first and second rows of Eq. (18), respectively, by setting $\Delta V_{c}=v_{c 1}-v_{c 2}, G_{0}^{\prime}=G_{0} L$, and $i_{\text {tot }}^{s}=i^{s} L$.

\section{Derivation of the IREE Length}

We start from the first row of Eq. (18), given by

$$
\Delta V_{c}=\left(\frac{L}{G_{B} \lambda}+\frac{2}{G_{0}^{\prime \prime}}\right) I_{c}-\frac{\alpha p_{0}}{2 G_{B}} i_{\text {tot }}^{s} .
$$

We assume that the channel resistance is much larger than the contact resistances, i.e., $L /\left(G_{B} \lambda\right) \gg 2 / G_{0}^{\prime \prime}$. This 


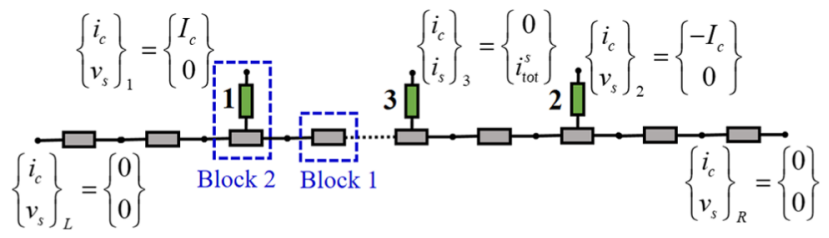

FIG. 6. SPICE setup for dc simulation. The setup connects the transmission-line circuit models in a distributed manner. Block 1 corresponds to the model in Fig. 1 and Block 2 corresponds to the model in Fig. 2. All the external contacts have $p_{f}=0$. All the terminals are two-component terminals: charge $(c)$ and $\operatorname{spin}(s)$. All indicated spin terminals are grounded except the spin terminal of contact 3 .

assumption yields

$$
\Delta V_{c}=\frac{L}{G_{B} \lambda} I_{c}-\frac{\alpha p_{0}}{2 G_{B}} i_{\mathrm{tot}}^{s}
$$

We apply the short-circuit condition $v_{c 1}=v_{c 2}$ at contacts 1 and 2, i.e., $\Delta V_{c}=0$. Thus, we get

$$
I_{c}=\frac{\alpha p_{0} \lambda}{2} \frac{i_{\mathrm{tot}}^{s}}{L}
$$

The IREE length is given by Eq. (21) as

$$
\lambda_{\mathrm{IREE}}=\frac{J_{c}}{J_{s}}=\frac{I_{c} / w}{i_{\mathrm{tot}}^{s} /(w L)}=\frac{\alpha p_{0} \lambda}{2},
$$

which is the expression in Eq. (22). Applying $\alpha=2 / \pi$ yields Eq. (23).

\section{APPENDIX B: SIMULATION SETUP}

This appendix provides the details of the simulation setup in SPICE that is used to analyze steady-state results of charge-spin interconversion in Sec. III.

We discretize the structure in Figs. 3(a), 3(c), and 4(a) into 100 small sections and represent each of the small sections with the corresponding circuit model. For example, block 1 and block 2 indicated in Fig. 6 are represented with the models in Figs. 1 and 2, respectively. Note that each of the nodes in Fig. 6 is a two-component node: charge $(c)$ and $z$ component of spin $(s)$. We connect the charge and spin terminals of the models for all the small sections in a modular fashion using standard circuit rules as shown in Fig. 6. We perform a dc simulation in SPICE. Note that during dc simulation in SPICE, capacitors and inductors automatically become open and short circuit, respectively, and correspond to the steady-state $(\partial / \partial t \rightarrow 0)$ form in Eq. (16).

The contacts $(1,2$, and 3$)$ in this discussion are point contacts. The contact polarizations $p_{f}=0$ and conductances are in the potentiometric limit with $G_{0} \approx 0.05 G_{B}$. We set the total number of modes $M+N$ in the channel to be 100 . We assume that the reflection with the spinflip scattering mechanism is dominant in the channel, i.e., $r_{s 1,2} \gg r, t_{s}$. The scattering rate per unit mode was set to 0.04 per lattice point.

We apply the charge open and spin ground boundary condition at the two boundaries given by

$$
\left\{\begin{array}{l}
i_{c} \\
v_{s}
\end{array}\right\}_{L}=\left\{\begin{array}{l}
0 \\
0
\end{array}\right\} \text { and }\left\{\begin{array}{l}
i_{c} \\
v_{s}
\end{array}\right\}_{R}=\left\{\begin{array}{l}
0 \\
0
\end{array}\right\} .
$$

Here, $i_{c}$ and $v_{s}$ indicate the boundary charge current and boundary spin voltage, respectively. Indices $L$ and $R$ indicate the left and right boundaries, respectively.

Setup in Fig. 3(a): For the setup in Fig. 3(a), we apply a current $I_{c}$ at the charge terminals of contacts 1 and 2, given by

$$
\left\{\begin{array}{l}
i_{c} \\
v_{s}
\end{array}\right\}_{1}=\left\{\begin{array}{c}
I_{c} \\
0
\end{array}\right\} \quad \text { and } \quad\left\{\begin{array}{c}
i_{c} \\
v_{s}
\end{array}\right\}_{2}=\left\{\begin{array}{c}
-I_{c} \\
0
\end{array}\right\}
$$

where indices 1 and 2 represent contacts 1 and 2, respectively. The spin terminals of contacts 1 and 2 are grounded to take into account the spin relaxation within the contact. Both charge and spin terminals of contact 3 are open and we observe open circuit spin voltage at the spin terminal. The boundary condition at contact 3 is

$$
\left\{\begin{array}{l}
i_{c} \\
i_{s}
\end{array}\right\}_{3}=\left\{\begin{array}{l}
0 \\
0
\end{array}\right\}
$$

where the index 3 indicates contact 3 .

Setup in Fig. 3(c): For the setup in Fig. 3(c), the charge terminal of contact 3 is open and we apply a current $i_{\text {tot }}^{s}$ at the spin terminal, given by

$$
\left\{\begin{array}{l}
i_{c} \\
i_{s}
\end{array}\right\}_{3}=\left\{\begin{array}{c}
0 \\
i_{\text {tot }}^{s}
\end{array}\right\}
$$

The spin terminals of contacts 1 and 2 are grounded to take into account the spin relaxation within the contact and charge terminals are kept open. Here, we observe the open circuit voltage difference between the charge terminals of contacts 1 and 2 . The boundary conditions are

$$
\left\{\begin{array}{l}
i_{c} \\
v_{s}
\end{array}\right\}_{1}=\left\{\begin{array}{l}
0 \\
0
\end{array}\right\} \text { and }\left\{\begin{array}{l}
i_{c} \\
v_{s}
\end{array}\right\}_{2}=\left\{\begin{array}{l}
0 \\
0
\end{array}\right\}
$$

Setup in Fig. 4(a): For the setup in Fig. 4(a), the charge terminal of contact 3 is open and we inject a current $i_{\text {tot }}^{s}$ through the spin terminal. The boundary condition at contact 3 is

$$
\left\{\begin{array}{l}
i_{c} \\
i_{s}
\end{array}\right\}_{3}=\left\{\begin{array}{c}
0 \\
i_{\text {tot }}^{s}
\end{array}\right\} .
$$

We short circuit the charge terminals of contacts 1 and 2 
TABLE II. Estimation of $k_{F}$.

\begin{tabular}{lcc}
\hline \hline Material & Electron density & $k_{F}\left(\mathrm{~nm}^{-1}\right)$ \\
\hline $\mathrm{Ag} / \mathrm{Bi}^{\mathrm{a}}$ & $5.86 \times 10^{28} \mathrm{~m}^{-3}[65]$ & 12 [Eq. (C1a)] \\
$\mathrm{Cu} / \mathrm{Bi}^{\mathrm{a}}$ & $8.49 \times 10^{28} \mathrm{~m}^{-3}[65]$ & 13.6 [Eq. (C1a)] \\
$\mathrm{LAO} / \mathrm{STO}$ & $2.6 \times 10^{17} \mathrm{~m}^{-2}[20]$ & 1.278 [Eq. (C1b)] \\
$\mathrm{Bi}_{2} \mathrm{Se}_{3}$ & $4 \times 10^{17} \mathrm{~m}^{-2}[42]$ & 1.59 [Eq. (C1b)] \\
\hline \hline
\end{tabular}

${ }^{\mathrm{a}}$ We use the electron densities of $\mathrm{Ag}$ and $\mathrm{Cu}$, respectively, as they are the most conductive layer in the corresponding bilayer.

and observe the short-circuit charge current $I_{c}$ flowing in the channel induced by $i_{\text {tot }}^{s}$. The spin terminals of contacts 1 and 2 are grounded to take into account the spin relaxation within the contact. The boundary conditions at contacts 1 and 2 are

$$
\left\{\begin{array}{l}
v_{c} \\
v_{s}
\end{array}\right\}_{1}=\left\{\begin{array}{c}
v_{c} \\
0
\end{array}\right\}_{2} \text { and }\left\{\begin{array}{c}
v_{c} \\
v_{s}
\end{array}\right\}_{2}=\left\{\begin{array}{c}
v_{c} \\
0
\end{array}\right\}_{1} .
$$

\section{APPENDIX C: PARAMETER ESTIMATIONS}

This appendix provides the details of the estimations made for the IREE lengths on diverse materials using Eq. (23).

\section{Estimations}

\section{Estimation of Fermi wave vector $\left(\boldsymbol{k}_{F}\right)$}

We estimate the Fermi wave vector $k_{F}$ of the channel from the electron density $n_{3 \mathrm{D}}$ (units of $\mathrm{m}^{-3}$ ) or $n_{2 \mathrm{D}}$ (units of $\mathrm{m}^{-2}$ ), using the following expressions:

$$
\begin{gathered}
k_{F}=\sqrt[3]{3 \pi^{2} n_{3 \mathrm{D}}} \\
k_{F}=\sqrt{2 \pi n_{2 \mathrm{D}}}
\end{gathered}
$$

Estimations are summarized in Table II.

\section{Estimation of ballistic conductance $\left(G_{B}\right)$}

We estimate the total number of modes in the threedimensional (3D) channel using the following expression:

$$
M+N=\frac{k_{F}^{2} w t}{2 \pi}
$$

TABLE IV. Estimation of $\lambda$.

\begin{tabular}{lccc}
\hline \hline Material & $R_{S}(\Omega / \square)$ & $\rho(\mu \Omega \mathrm{cm})$ & $\lambda(\mathrm{nm})$ \\
\hline $\mathrm{Ag} / \mathrm{Bi}$ & $10^{\mathrm{a}}$ & $\ldots$ & 22.6 [Eq. (C5)] \\
$\mathrm{Cu} / \mathrm{Bi}$ & $\cdots$ & $100^{\mathrm{c}}$ & 0.88 [Eq. (C6)] \\
$\mathrm{LAO} / \mathrm{STO}$ & $176^{\mathrm{b}}$ & $\cdots$ & 180.8 [Eq. (C5)] \\
$\mathrm{Bi}_{2} \mathrm{Se}_{3}$ & $\cdots$ & $2000^{\mathrm{d}}$ & 3.2 [Eq. (C6)] \\
\hline \hline
\end{tabular}

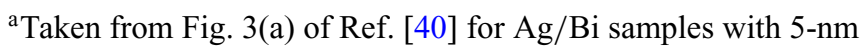
Ag.

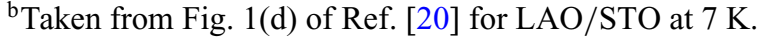

${ }^{\mathrm{c}} \rho$ of $\mathrm{Bi}$ layer is used, which is taken from Ref. [41].

${ }^{\mathrm{d}}$ Taken from Fig. 2(b) of Ref. [42] at approximately $300 \mathrm{~K}$.

where $w$ is the width and $t$ is the thickness. For a 2D channel, the expression is

$$
M+N=\frac{2 k_{F} w}{\pi} .
$$

Note that, in a strong TI 2D channel with limited bulk conduction, $M+N$ will be lower by a factor of 2 than Eq. (C3). We estimate the ballistic conductance of the channel using the following expression:

$$
G_{B}=\frac{q^{2}}{h}(M+N) .
$$

Estimations are summarized in Table III.

\section{Estimation of mean free path $(\lambda)$}

The mean free path $(\lambda)$ is estimated from the measured sheet resistance $R_{S}$ of the sample using the expression

$$
\frac{R_{S}}{w}=\frac{1}{G_{B} \lambda},
$$

or from the resistivity $\rho$ of the sample using the expression

$$
\frac{\rho}{w t}=\frac{1}{G_{B} \lambda} .
$$

Estimations are summarized in Table IV.

TABLE III. Estimation of $G_{B}$.

\begin{tabular}{lcccc}
\hline \hline Material & $w(\mu \mathrm{m})$ & $t(\mathrm{~nm})$ & $M+N$ & $G_{B}$ [Eq. (C4)] \\
\hline $\mathrm{Ag} / \mathrm{Bi}^{\mathrm{a}}$ & $400[40]$ & $5[40]$ & $4.6 \times 10^{7}$ [Eq. (C2)] & $1.77 \mathrm{kS}$ \\
$\mathrm{Cu} / \mathrm{Bi}^{\mathrm{a}}$ & $0.15[41]$ & $20[41]$ & $8.8 \times 10^{4}$ [Eq. (C2)] & $3.4 \mathrm{~S}$ \\
$\mathrm{LAO} / \mathrm{STO}$ & $400[20]$ & $\cdots$ & $3.3 \times 10^{5}$ [Eq. (C3)] & $12.6 \mathrm{~S}$ \\
$\mathrm{Bi}_{2} \mathrm{Se}_{3}$ & $1000[42]$ & $9^{\mathrm{b}}[42]$ & $3.6 \times 10^{6}$ (Eq. (C2)) & $140 \mathrm{~S}$ \\
\hline \hline
\end{tabular}

${ }^{\mathrm{a}}$ We use the thicknesses of the most conductive layer (Ag and $\mathrm{Cu}$, respectively) to estimate $G_{B}$ considering bulk conduction.

${ }^{\mathrm{b}}$ We considered six quintuple-layer (QL) samples in Ref. [42]. $1 \mathrm{QL} \approx 1.5 \mathrm{~nm}$. 
TABLE V. Estimation of $p_{0}$.

\begin{tabular}{|c|c|c|c|}
\hline Material & $\alpha_{R}(\mathrm{eV} \AA)$ & $v_{F}\left(\times 10^{6} \mathrm{~m} \mathrm{~s}^{-1}\right)$ & $p_{0}$ \\
\hline $\mathrm{Ag} / \mathrm{Bi}$ & $0.56[40]$ & $\begin{array}{l}1.39(\mathrm{Ag})[65] \\
1.87(\mathrm{Bi})[65]\end{array}$ & 0.05 \\
\hline $\mathrm{Cu} / \mathrm{Bi}$ & $0.56[41]$ & $\begin{array}{l}1.57(\mathrm{Cu})[65] \\
1.87(\mathrm{Bi})[65]\end{array}$ & 0.05 \\
\hline $\mathrm{LAO} / \mathrm{STO}$ & 0.03 [20] & $0.074^{\mathrm{a}}$ & 0.0616 \\
\hline $\begin{array}{l}\text { Material } \\
\mathrm{Bi}_{2} \mathrm{Se}_{3}\end{array}$ & $\begin{array}{c}\Delta V_{c}(\mu \mathrm{V}) \\
40^{\mathrm{b}}[42]\end{array}$ & $\begin{array}{l}J_{s}\left(\mathrm{~A} \mathrm{~m}^{-2}\right) \\
1.39 \times 10^{5 \mathrm{c}}\end{array}$ & $\begin{array}{c}p_{0} \\
0.025 \\
\text { (Eq. (20)). }\end{array}$ \\
\hline
\end{tabular}

${ }^{a}$ We estimate the Fermi velocity using $v_{F}=\hbar k_{F} / m^{*} . m^{*} \approx 2$ $\times 9.1 \times 10^{-31} \mathrm{~kg}$ as reported in Ref. [20].

${ }^{\mathrm{b}}$ Inverse-effect-induced voltage due to spin pumping at $3 \mathrm{GHz}$. ${ }^{\mathrm{c}}$ Taken from table 1 in supplementary information of Ref. [42] for $6 \mathrm{QL}$. The sample dimension is $1 \mathrm{~mm} \times 5 \mathrm{~mm}$ [42], which yields $i_{\text {tot }}^{s} \approx 0.695 \mathrm{~A}$.

\section{Estimation of degree of spin-momentum locking $\left(p_{\mathbf{0}}\right)$}

For $\mathrm{Ag} / \mathrm{Bi}, \mathrm{Cu} / \mathrm{Bi}$, and $\mathrm{LAO} / \mathrm{STO}$, the degree of SML $p_{0}$ is estimated using the Rashba coupling coefficient $\left(v_{0}=\right.$ $\left.\alpha_{R} / \hbar\right)$ and the Fermi velocity $\left(v_{F}\right)$ of the materials, using Eq. (24). For $\mathrm{Bi}_{2} \mathrm{Se}_{3}, p_{0}$ is estimated using spin current density reported from spin pumping $\left(J_{s}\right)$, the measured inverse effect voltage $\Delta V_{c}$, and Eq. (20). The estimations are summarized in Table $\mathrm{V}$.

\section{Derivation of Eq. (24)}

We start from the eigenstates in Eq. (37) of the Rashba Hamiltonian in Eq. (36), given by

$$
E=\frac{p^{\prime 2}}{2 m}-s v_{0} p^{\prime}+U_{E},
$$

with $p^{\prime}=|\vec{p}-q \vec{A}|$. Solutions for $p^{\prime}$ are given by

$$
\begin{aligned}
& p^{\prime}{ }_{1}(s)=s m v_{0}+\sqrt{m^{2} v_{0}^{2}+2 m\left(E-U_{E}\right)}, \\
& p^{\prime}{ }_{2}(s)=s m v_{0}-\sqrt{m^{2} v_{0}^{2}+2 m\left(E-U_{E}\right)},
\end{aligned}
$$

noting that $s^{2}=1$. Here, $p^{\prime}{ }_{1}(s=+1)$ and $p^{\prime}{ }_{1}(s=-1)$ correspond to $M$ and $N$, respectively. Similarly, $p_{2}^{\prime}(s=$ $-1)$ and $p_{2}^{\prime}(s=+1)$ correspond to $M$ and $N$, respectively. Thus, the degree of SML $p_{0}$ is given by

$$
\begin{aligned}
p_{0}\left(E_{F}\right) & =\frac{p^{\prime}{ }_{1}(s=+1)-p^{\prime}{ }_{1}(s=-1)}{p^{\prime}{ }_{1}(s=+1)+p^{\prime}{ }_{1}(s=-1)} \\
& =\frac{v_{0}}{\sqrt{v_{0}^{2}+\frac{2\left(E_{F}-U_{E}\right)}{m}}} .
\end{aligned}
$$

Assuming $U_{E}=0$ and applying $E_{F}=\frac{1}{2} m v_{F}^{2}$, we have the expression in Eq. (24).

\section{APPENDIX D: CHARGE AND SPIN VELOCITIES}

This appendix provides the derivation of eigenvalues and eigenvectors of Eqs. (2) and (3) to find the charge and spin velocities and their coupling during propagation.

The matrix form of Eqs. (2) and (3) is given by

$$
\begin{gathered}
{\left[\begin{array}{cccc}
C_{\mathrm{eff}} & 0 & 0 & 0 \\
0 & \frac{C_{Q}}{\alpha^{2}} & p_{0} g_{m} L_{M} & 0 \\
0 & 0 & L_{\mathrm{eff}} & 0 \\
-p_{0} r_{m} C_{\mathrm{eff}} & 0 & 0 & \alpha^{2} L_{K}
\end{array}\right] \frac{\partial}{\partial t}\left\{\begin{array}{l}
V_{c} \\
V_{s} \\
I_{c} \\
I_{s}
\end{array}\right\}} \\
+\left[\begin{array}{cccc}
0 & 0 & 0 & 0 \\
0 & G_{\mathrm{sh}} & -p_{0} \gamma_{s} & 0 \\
0 & -p_{0} \eta_{c} & R_{c} & 0 \\
-p_{0} \eta_{s} & 0 & 0 & R_{s}
\end{array}\right]\left\{\begin{array}{l}
V_{c} \\
V_{s} \\
I_{c} \\
I_{s}
\end{array}\right\} \\
=-\left[\begin{array}{cccc}
0 & 0 & 1 & 0 \\
0 & 0 & 0 & 1 \\
1 & 0 & 0 & 0 \\
0 & 1 & 0 & 0
\end{array}\right] \frac{\partial}{\partial x}\left\{\begin{array}{c}
V_{c} \\
V_{s} \\
I_{c} \\
I_{s}
\end{array}\right\} .
\end{gathered}
$$

In the low-loss limit, we assume a solution of the form

$$
\left\{\begin{array}{l}
V_{c} \\
V_{s} \\
I_{c} \\
I_{s}
\end{array}\right\} \equiv\left\{\begin{array}{l}
\tilde{V}_{c} \\
\tilde{V}_{s} \\
\tilde{I}_{c} \\
\tilde{I}_{s}
\end{array}\right\} e^{j(k x-\omega t)}
$$

which results in

$$
\begin{gathered}
-j \omega\left[\begin{array}{cccc}
C_{\mathrm{eff}} & 0 & 0 & 0 \\
0 & \frac{C_{Q}}{\alpha^{2}} & p_{0} g_{m} L_{M} & 0 \\
0 & 0 & L_{\mathrm{eff}} & 0 \\
-p_{0} r_{m} C_{\mathrm{eff}} & 0 & 0 & \alpha^{2} L_{K}
\end{array}\right]\left\{\begin{array}{l}
\tilde{V}_{c} \\
\tilde{V}_{s} \\
\tilde{I}_{c} \\
\tilde{I}_{s}
\end{array}\right\} \\
+\left[\begin{array}{cccc}
0 & 0 & 0 & 0 \\
0 & G_{\mathrm{sh}} & -p_{0} \gamma_{s} & 0 \\
0 & -p_{0} \eta_{c} & R_{c} & 0 \\
-p_{0} \eta_{s} & 0 & 0 & R_{s}
\end{array}\right]\left\{\begin{array}{l}
\tilde{V}_{c} \\
\tilde{V}_{s} \\
\tilde{I}_{c} \\
\tilde{I}_{s}
\end{array}\right\} \\
=-j k\left[\begin{array}{cccc}
0 & 0 & 1 & 0 \\
0 & 0 & 0 & 1 \\
1 & 0 & 0 & 0 \\
0 & 1 & 0 & 0
\end{array}\right]\left\{\begin{array}{l}
\tilde{V}_{c} \\
\tilde{V}_{s} \\
\tilde{I}_{c} \\
\tilde{I}_{s}
\end{array}\right\} .
\end{gathered}
$$

We assume that the coefficients of the transmission-line model [Eqs. (2) and (3)] are constant with frequency $\omega$ and propagation vector $k$. We differentiate both sides of Eq. (D3) with respect to $k$ and the following matrix 
equation:

$$
v_{g}\left\{\begin{array}{c}
\tilde{V}_{c} \\
\tilde{V}_{s} \\
\tilde{I}_{c} \\
\tilde{I}_{s}
\end{array}\right\}=\left[\begin{array}{cccc}
0 & 0 & \frac{1}{C_{\mathrm{eff}}} & 0 \\
-\frac{\alpha^{2} p_{0} g_{m} L_{M}}{C_{Q} L_{\mathrm{eff}}} & 0 & 0 & \frac{\alpha^{2}}{C_{Q}} \\
\frac{1}{L_{\mathrm{eff}}} & 0 & 0 & 0 \\
0 & \frac{1}{\alpha^{2} L_{K}} & \frac{p_{0} r_{m}}{\alpha^{2} L_{K}} & 0
\end{array}\right]\left\{\begin{array}{c}
\tilde{V}_{c} \\
\tilde{V}_{s} \\
\tilde{I}_{c} \\
\tilde{I}_{s}
\end{array}\right\},
$$

where $v_{g}$ is the group velocity given by

$$
v_{g}=\frac{\partial \omega}{\partial k} .
$$

Note that the eigenvalues of Eq. (D4) give the velocities in Eqs. (25) and (27). For a particular eigenvalue $v_{g}$, we can write the following equation from Eq. (D4):

$$
\begin{aligned}
& {\left[\begin{array}{cc}
-v_{g} & \frac{1}{C_{\mathrm{eff}}} \\
\frac{1}{L_{\mathrm{eff}}} & -v_{g}
\end{array}\right]\left\{\begin{array}{l}
\tilde{V}_{c} \\
\tilde{I}_{c}
\end{array}\right\}=\left\{\begin{array}{l}
0 \\
0
\end{array}\right\}} \\
& {\left[\begin{array}{cc}
-v_{g} & \frac{\alpha^{2}}{C_{Q}} \\
\frac{1}{\alpha^{2} L_{K}} & -v_{g}
\end{array}\right]\left\{\begin{array}{l}
\tilde{V}_{s} \\
\tilde{I}_{s}
\end{array}\right\}=p_{0}\left\{\begin{array}{c}
\frac{\alpha^{2} g_{m} L_{M}}{C_{Q} L_{\mathrm{eff}}} \tilde{V}_{c} \\
-\frac{r_{m}}{\alpha^{2} L_{K}} \tilde{I}_{c}
\end{array}\right\} \text {. }}
\end{aligned}
$$

For the eigenvalue $v_{g}=v_{g, s}= \pm 1 / \sqrt{L_{K} C_{Q}}$ in Eq. (D6), we have $\tilde{V}_{c}=\tilde{I}_{c}=0$ and, assuming $\tilde{I}_{s}=1$, we get Eq. (26). Again, for the eigenvalue $v_{g}=v_{g, c}= \pm 1 / \sqrt{L_{\mathrm{eff}} C_{\mathrm{eff}}}$, we get Eq. (28), assuming $\tilde{I}_{c}=1$.

\section{APPENDIX E: DERIVATION OF SEMICLASSICAL MODEL}

This appendix provides the derivation of Eq. (35), starting from the Boltzmann transport equation in Eq. (31).

We apply a variable transformation $\xi(x, t, \vec{p}, \vec{s}) \equiv$ $E(x, t, \vec{p}, \vec{s})-\mu(x, t, \vec{p}, \vec{s})$ on the left hand side of Eq. (31), which yields

$$
\begin{aligned}
& \frac{\partial f}{\partial t}=\frac{\partial f}{\partial \xi}\left(\frac{\partial E}{\partial t}-\frac{\partial \mu}{\partial t}\right), \\
& v_{x} \frac{\partial f}{\partial x}=v_{x} \frac{\partial f}{\partial \xi}\left(\frac{\partial E}{\partial x}-\frac{\partial \mu}{\partial x}\right), \text { and } \\
& F_{x} \frac{\partial f}{\partial p_{x}}=F_{x} \frac{\partial f}{\partial \xi}\left(\frac{\partial E}{\partial p_{x}}-\frac{\partial \mu}{\partial p_{x}}\right) .
\end{aligned}
$$

We first substitute $F_{x}=-\partial E / \partial x$ and $v_{x}=\partial E / \partial p_{x}$. Finally, we set $\partial f / \partial \xi=\partial f_{0} / \partial E$ to get the left-hand side of Eq. (34).
On the right-hand side of Eq. (31), we expand both $f$ and $f^{\prime}$ into Taylor series around $f_{0}$ [Eq. (33)] with constant electrochemical potential $\mu_{0}$. We set $\xi^{\prime}\left(x, t, \vec{p}^{\prime}, s^{\prime}\right) \equiv$ $E\left(x, t, \vec{p}^{\prime}, s^{\prime}\right)-\mu\left(x, t, \vec{p}^{\prime}, s^{\prime}\right)$ and $\xi_{0}(x, \vec{p}, s) \equiv E(x, \vec{p}, s)-$ $\mu_{0}$ and assume that $\xi$ and $\xi^{\prime}$ are close to $\xi_{0}$, which gives

$$
\begin{aligned}
& f \approx f_{0}+\left(-\frac{\partial f}{\partial \xi}\right)_{\xi=\xi_{0}}\left(\xi-\xi_{0}\right) \quad \text { and } \\
& f^{\prime} \approx f_{0}+\left(-\frac{\partial f}{\partial \xi^{\prime}}\right)_{\xi^{\prime}=\xi_{0}}\left(\xi^{\prime}-\xi_{0}\right) .
\end{aligned}
$$

We set $(\partial f / \partial \xi)_{\xi=\xi_{0}}=\left(\partial f / \partial \xi^{\prime}\right)_{\xi^{\prime}=\xi_{0}}=\partial f_{0} / \partial E$. Thus, the right-hand side of Eq. (31) becomes

$$
f-f^{\prime}=\left(-\frac{\partial f_{0}}{\partial E}\right)\left(\xi-\xi^{\prime}\right)=\left(\frac{\partial f_{0}}{\partial E}\right)\left(\mu-\mu^{\prime}\right),
$$

noting that $E\left(x, t, \vec{p}^{\prime}, s^{\prime}\right)=E(x, t, \vec{p}, s)$ in the elastic scattering limit.

\section{APPENDIX F: E-P RELATION}

This appendix provides the derivation of Eq. (39), starting from the $E-p$ relation in Eq. (38).

Differentiating Eq. (38) with respect to time $t$ yields

$$
\frac{\partial E}{\partial t}=\left(\frac{\vec{p}-q \vec{A}}{m}-s v_{0} \frac{\vec{p}-q \vec{A}}{|\vec{p}-q \vec{A}|}\right) \cdot\left(-q \frac{\partial \vec{A}}{\partial t}\right)+\frac{\partial U_{E}}{\partial t} .
$$

The velocity $\vec{v}(E)=\nabla_{\vec{p}} E$ is derived from Eq. (38) as

$$
\vec{v}(E)=\frac{\vec{p}-q \vec{A}}{m}-s v_{0} \frac{\vec{p}-q \vec{A}}{|\vec{p}-q \vec{A}|} .
$$

Combining Eqs. (F1) and (F2) yields Eq. (39).

Note that Eq. (F2) can be written as

$$
\vec{v}(E)=\left(\frac{|\vec{p}-q \vec{A}|}{m}+s v_{0}\right) \frac{\vec{p}-q \vec{A}}{|\vec{p}-q \vec{A}|},
$$

where $(\vec{p}-q \vec{A}) /|\vec{p}-q \vec{A}|$ is a unit vector along $\vec{p}-q \vec{A}$. From Eq. (38), we get

$$
|\vec{p}-q \vec{A}|=-s m v_{0} \pm m \sqrt{v_{0}^{2}+\frac{2\left(E-U_{E}\right)}{m}},
$$

noting that $s^{2}=1$. Thus, from Eq. (F3), we get

$$
\vec{v}(E)= \pm \sqrt{v_{0}^{2}+\frac{2\left(E-U_{E}\right)}{m}} \frac{\vec{p}-q \vec{A}}{|\vec{p}-q \vec{A}|} .
$$

Note that the magnitude of the velocity for a particular energy of interest is the same for all four groups in Eq. (44) for the Rashba Hamiltonian in Eq. (36) considered here. 


\section{APPENDIX G: SCATTERING RATES}

This appendix provides the derivation of the scattering matrix in Eq. (56).

For the group $\Re \equiv U^{+}, D^{-}, U^{-}$, and $D^{+}$, we have from Eq. (54)

$$
\begin{aligned}
\tilde{S}\left(U^{+}\right)= & \hat{S}_{U^{+} \leftrightarrow D^{-}}\left[\left\langle\mu\left(D^{-}\right)\right\rangle-\left\langle\mu\left(U^{+}\right)\right\rangle\right] \\
& +\hat{S}_{U^{+} \leftrightarrow U^{-}}\left[\left\langle\mu\left(U^{-}\right)\right\rangle-\left\langle\mu\left(U^{+}\right)\right\rangle\right] \\
& +\hat{S}_{U^{+} \leftrightarrow D^{+}}\left[\left\langle\mu\left(D^{+}\right)\right\rangle-\left\langle\mu\left(U^{+}\right)\right\rangle\right], \\
\tilde{S}\left(D^{-}\right)= & \hat{S}_{D^{-} \leftrightarrow U^{+}}\left[\left\langle\mu\left(U^{+}\right)\right\rangle-\left\langle\mu\left(D^{-}\right)\right\rangle\right] \\
& +\hat{S}_{D^{-} \leftrightarrow U^{-}}\left[\left\langle\mu\left(U^{-}\right)\right\rangle-\left\langle\mu\left(D^{-}\right)\right\rangle\right] \\
& +\hat{S}_{D^{-} \leftrightarrow D^{+}}\left[\left\langle\mu\left(D^{+}\right)\right\rangle-\left\langle\mu\left(D^{-}\right)\right\rangle\right], \\
\tilde{S}\left(U^{-}\right)= & \hat{S}_{U^{-} \leftrightarrow U^{+}}\left[\left\langle\mu\left(U^{+}\right)\right\rangle-\left\langle\mu\left(U^{-}\right)\right\rangle\right] \\
& +\hat{S}_{U^{-} \leftrightarrow D^{-}}\left[\left\langle\mu\left(D^{-}\right)\right\rangle-\left\langle\mu\left(U^{-}\right)\right\rangle\right] \\
& +\hat{S}_{U^{-} \leftrightarrow D^{+}}\left[\left\langle\mu\left(D^{+}\right)\right\rangle-\left\langle\mu\left(U^{-}\right)\right\rangle\right],
\end{aligned}
$$

and

$$
\begin{aligned}
\tilde{S}\left(D^{+}\right)= & \hat{S}_{D^{+} \leftrightarrow U^{+}}\left[\left\langle\mu\left(U^{+}\right)\right\rangle-\left\langle\mu\left(D^{+}\right)\right\rangle\right] \\
& +\hat{S}_{D^{+} \leftrightarrow D^{-}}\left[\left\langle\mu\left(D^{-}\right)\right\rangle-\left\langle\mu\left(D^{+}\right)\right\rangle\right] \\
& +\hat{S}_{D^{+} \leftrightarrow U^{-}}\left[\left\langle\mu\left(U^{-}\right)\right\rangle-\left\langle\mu\left(D^{+}\right)\right\rangle\right] .
\end{aligned}
$$

Equations (G1), (G2), (G3), and (G4) together yield the scattering matrix in Eq. (56) in the $\left\{\mu\left(U^{+}\right), \mu\left(D^{-}\right), \mu\left(U^{-}\right)\right.$, $\left.\mu\left(D^{+}\right)\right\}^{T}$ basis.

\section{APPENDIX H: CHARGE AND SPIN CURRENTS AND VOLTAGES}

This appendix provides the derivation of Eq. (61).

The current in any group is given by

$$
I(\Re)=\frac{q}{L} \sum_{\vec{p}, s \in \Re} v_{x} f(x, t, \vec{p}, s),
$$

where $f$ is given by Eq. (32). Under the linear response approximation, we can write

$$
f(x, t, \vec{p}, s) \approx f_{0}+\left(-\frac{\partial f_{0}}{\partial E}\right)\left[\mu(x, t, \vec{p}, s)-\mu_{0}\right],
$$

where $f_{0}$ is given by Eq. (33) with constant electrochemical potential $\mu_{0}$. Thus, from Eq. (H1), we can write

$I(\Re)=\frac{q}{L}\left[f_{0} \sum_{\vec{p}, s \in \Re} v_{x}+\frac{D_{0}(\Re)}{2}\left(\left\langle v_{x} \mu\right\rangle_{\vec{p}, s \in \Re}-\mu_{0}\left\langle v_{x}\right\rangle\right)\right]$, where $D_{0}(\Re)$ is given by Eq. (47) and the averaging is defined by Eq. (45).

We use the assumption in Eq. (48), given by

$$
\left\langle v_{x} \mu\right\rangle_{\vec{p}, s \in \Re} \approx\left\langle v_{x}\right\rangle\langle\mu\rangle_{\vec{p}, s \in \Re},
$$

which results in

$$
\begin{aligned}
I(\Re)= & \operatorname{sgn}\left(\left\langle v_{x}\right\rangle\right) \frac{q}{h} n_{m}(\Re)\left(\langle\mu\rangle_{\vec{p}, s \in \Re}-\mu_{0}\right) \\
& +\operatorname{sgn}\left(\left|v_{x}\right|\right) \frac{q}{L} f_{0} \sum_{\vec{p}, s \in \Re}\left|v_{x}\right|,
\end{aligned}
$$

where $n_{m}(\Re)$ is given by Eq. (50).

\section{Charge current}

The charge current in the channel is given by

$$
I_{c}=I\left(U^{+}\right)+I\left(D^{+}\right)+I\left(U^{-}\right)+I\left(D^{-}\right),
$$

which in conjunction with Eq. (H4) yields

$$
\begin{aligned}
I_{c}= & \frac{q}{h}\left\{\frac{h}{L} f_{0} \sum_{\vec{p}, s \in U^{+}}\left|v_{x}\right|+M\left[\mu\left(U^{+}\right)-\mu_{0}\right]\right\} \\
& +\frac{q}{h}\left\{\frac{h}{L} f_{0} \sum_{\vec{p}, s \in D^{+}}\left|v_{x}\right|+N\left[\mu\left(D^{+}\right)-\mu_{0}\right]\right\} \\
& -\frac{q}{h}\left\{\frac{h}{L} f_{0} \sum_{\vec{p}, s \in U^{-}}\left|v_{x}\right|+N\left[\mu\left(U^{-}\right)-\mu_{0}\right]\right\} \\
& -\frac{q}{h}\left\{\frac{h}{L} f_{0} \sum_{\vec{p}, s \in D^{-}}\left|v_{x}\right|+M\left[\mu\left(D^{-}\right)-\mu_{0}\right]\right\},
\end{aligned}
$$

where $\quad n_{m}\left(U^{+}\right)=n_{m}\left(D^{-}\right)=M \quad$ and $\quad n_{m}\left(U^{-}\right)=$ $n_{m}\left(D^{+}\right)=N$.

Note that $\left(U^{+}, D^{-}\right)$and $\left(U^{-}, D^{+}\right)$are time-reversal symmetric pairs; hence,

$$
\text { and } \begin{aligned}
\sum_{\vec{p}, s \in U^{+}}\left|v_{x}\right| & =\sum_{\vec{p}, s \in D^{-}}\left|v_{x}\right| \\
\sum_{\vec{p}, s \in U^{-}}\left|v_{x}\right| & =\sum_{\vec{p}, s \in D^{+}}\left|v_{x}\right|,
\end{aligned}
$$

which, when applied to Eq. (H6), give the expression for the charge current

$I_{c}=\frac{q}{h}\left[M \tilde{\mu}\left(U^{+}\right)-N \tilde{\mu}\left(U^{-}\right)+N \tilde{\mu}\left(D^{+}\right)-M \tilde{\mu}\left(D^{-}\right)\right]$,

where we defined $\tilde{\mu}=\mu-\mu_{0}$. 


\section{Spin current}

The spin current in the channel is given by

$$
\tilde{I}_{s}=\frac{1}{\alpha}\left[I\left(U^{+}\right)+I\left(U^{-}\right)-I\left(D^{+}\right)-I\left(D^{-}\right)\right],
$$

where $\alpha=2 / \pi$ is an angular averaging factor to take into account the average $\hat{z} \cdot \vec{s}$ on a half Fermi circle [see Fig. 5(a)]. Equation (H9) in conjunction with Eq. (H4) yields

$$
\begin{aligned}
\tilde{I}_{s}= & \frac{1}{\alpha} \frac{q}{h}\left\{\frac{h}{L} f_{0} \sum_{\vec{p}, s \in U^{+}}\left|v_{x}\right|+M\left[\mu\left(U^{+}\right)-\mu_{0}\right]\right\} \\
& -\frac{1}{\alpha} \frac{q}{h}\left\{\frac{h}{L} f_{0} \sum_{\vec{p}, s \in U^{-}}\left|v_{x}\right|+N\left[\mu\left(U^{-}\right)-\mu_{0}\right]\right\} \\
& -\frac{1}{\alpha} \frac{q}{h}\left\{\frac{h}{L} f_{0} \sum_{\vec{p}, s \in D^{+}}\left|v_{x}\right|+N\left[\mu\left(D^{+}\right)-\mu_{0}\right]\right\} \\
& +\frac{1}{\alpha} \frac{q}{h}\left\{\frac{h}{L} f_{0} \sum_{\vec{p}, s \in D^{-}}\left|v_{x}\right|+M\left[\mu\left(D^{-}\right)-\mu_{0}\right]\right\} .
\end{aligned}
$$

Applying the condition in Eq. (H7), we have the expression for channel spin current as

$$
\begin{aligned}
\tilde{I}_{s}= & \frac{1}{\alpha} \frac{q}{h}\left[M \tilde{\mu}\left(U^{+}\right)-N \tilde{\mu}\left(U^{-}\right)-N \tilde{\mu}\left(D^{+}\right)+M \tilde{\mu}\left(D^{-}\right)\right] \\
& +\frac{2}{\alpha} \frac{q}{L} f_{0}\left(\sum_{\vec{p}, s \in U^{+}}\left|v_{x}\right|-\sum_{\vec{p}, s \in U^{-}}\left|v_{x}\right|\right) .
\end{aligned}
$$

Note that the first term is zero at the equilibrium condition $\mu\left(U^{+}\right)=\mu\left(U^{-}\right)=\mu\left(D^{+}\right)=\mu\left(D^{-}\right)=\mu_{0}$.

However, the second term is nonzero even at equilibrium since

$$
\sum_{\vec{p}, s \in U^{+}}\left|v_{x}\right| \neq \sum_{\vec{p}, s \in U^{-}}\left|v_{x}\right|
$$

and represents the equilibrium spin current in the channel. We subtract the equilibrium part from our definition of spin current, given by

$$
I_{s}=\frac{1}{\alpha} \frac{q}{h}\left[M \tilde{\mu}\left(U^{+}\right)-N \tilde{\mu}\left(U^{-}\right)-N \tilde{\mu}\left(D^{+}\right)+M \tilde{\mu}\left(D^{-}\right)\right] .
$$

\section{Spin voltage}

The spin voltage in the channel is given by

$$
q V_{s}=\alpha \frac{M \mu\left(U^{+}\right)+N \mu\left(U^{-}\right)-N \mu\left(D^{+}\right)-M \mu\left(D^{-}\right)}{2(M+N)} .
$$

Subtracting each electrochemical potential by $\mu_{0}$ gives

$$
q V_{s}=\alpha \frac{M \tilde{\mu}\left(U^{+}\right)+N \tilde{\mu}\left(U^{-}\right)-N \tilde{\mu}\left(D^{+}\right)-M \tilde{\mu}\left(D^{-}\right)}{2(M+N)} .
$$

\section{Charge voltage}

The charge voltage in the channel is given by

$$
q \tilde{V}^{c}=\frac{M \mu\left(U^{+}\right)+N \mu\left(U^{-}\right)+N \mu\left(D^{+}\right)+M \mu\left(D^{-}\right)}{2(M+N)} .
$$

We subtract each electrochemical potential by $\mu_{0}$ according to

$$
q V^{c}=\frac{M \tilde{\mu}\left(U^{+}\right)+N \tilde{\mu}\left(U^{-}\right)+N \tilde{\mu}\left(D^{+}\right)+M \tilde{\mu}\left(D^{-}\right)}{2(M+N)},
$$

where $V_{c}=\tilde{V}_{c}-\mu_{0} / q$.

Equations (H8), (H12), (H14), and (H16) together can be written in a matrix form given by Eq. (61).

[1] M. Z. Hasan and C. L. Kane, Colloquium: Topological insulators, Rev. Mod. Phys. 82, 3045 (2010).

[2] Xiao-Liang Qi and Shou-Cheng Zhang, Topological insulators and superconductors, Rev. Mod. Phys. 83, 1057 (2011).

[3] C. H. Li, O. M. van't Erve, J. T. Robinson, Y. Liu, L. Li, and B. T. Jonker, Electrical detection of charge-current-induced spin polarization due to spin-momentum locking in $\mathrm{Bi}_{2} \mathrm{Se}_{3}$, Nat. Nanotechnol. 9, 20325 (2014).

[4] Jianshi Tang, Li-Te Chang, Xufeng Kou, Koichi Murata, Eun Sang Choi, Murong Lang, Yabin Fan, Ying Jiang, Mohammad Montazeri, Wanjun Jiang, Yong Wang, Liang $\mathrm{He}$, and Kang L. Wang, Electrical detection of spinpolarized surface states conduction in (Bi0.53Sb0.47)2 $\mathrm{Te} 3$ topological insulator, Nano Lett. 14, 5423 (2014).

[5] André Dankert, Johannes Geurs, M. Venkata Kamalakar, Sophie Charpentier, and Saroj P. Dash, Room temperature electrical detection of spin polarized currents in topological insulators, Nano Lett. 15, 7976 (2015).

[6] Luqiao Liu, A. Richardella, Ion Garate, Yu Zhu, N. Samarth, and Ching-Tzu Chen, Spin-polarized tunneling study of spin-momentum locking in topological insulators, Phys. Rev. B 91, 235437 (2015).

[7] Jifa Tian, Ireneusz Miotkowski, Seokmin Hong, and Yong P. Chen, Electrical injection and detection of spin-polarized 
currents in topological insulator $\mathrm{Bi}_{2} \mathrm{Te}_{2} \mathrm{Se}$, Sci. Rep. 5, 14293 (2015).

[8] Joon Sue Lee, Anthony Richardella, Danielle Reifsnyder Hickey, K. Andre Mkhoyan, and Nitin Samarth, Mapping the chemical potential dependence of current-induced spin polarization in a topological insulator, Phys. Rev. B 92, 155312 (2015).

[9] Fan Yang, Subhamoy Ghatak, A. A. Taskin, Kouji Segawa, Yuichiro Ando, Masashi Shiraishi, Yasushi Kanai, Kazuhiko Matsumoto, Achim Rosch, and Yoichi Ando, Switching of charge-current-induced spin polarization in the topological insulator BiSbTeSe 2 , Phys. Rev. B 94, 075304 (2016).

[10] Jehyun Kim, Chaun Jang, Xiangfeng Wang, Johnpierre Paglione, Seokmin Hong, and Dohun Kim, Electrical detection of surface spin polarization of candidate topological kondo insulator smb6, arXiv:1809.04977 [cond-mat.str-el] (2018).

[11] Ioan Mihai Miron, Kevin Garello, Gilles Gaudin, PierreJean Zermatten, Marius V. Costache, Stephane Auffret, Sebastien Bandiera, Bernard Rodmacq, Alain Schuhl, and Pietro Gambardella, Perpendicular switching of a single ferromagnetic layer induced by in-plane current injection, Nature 476, 189 (2011).

[12] T. Suzuki, S. Fukami, N. Ishiwata, M. Yamanouchi, S. Ikeda, N. Kasai, and H. Ohno, Current-induced effective field in perpendicularly magnetized $\mathrm{Ta} / \mathrm{CoFeB} / \mathrm{MgO}$ wire, Appl. Phys. Lett. 98, 142505 (2011).

[13] Chi-Feng Pai, Luqiao Liu, Y. Li, H. W. Tseng, D. C. Ralph, and R. A. Buhrman, Spin transfer torque devices utilizing the giant spin hall effect of tungsten, Appl. Phys. Lett. 101, 122404 (2012).

[14] Luqiao Liu, Chi-Feng Pai, Y. Li, H. W. Tseng, D. C. Ralph, and R. A. Buhrman, Spin-torque switching with the giant spin hall effect of tantalum, Science 336, 555 (2012).

[15] Binghai Yan, Benjamin Stadtmüller, Norman Haag, Sebastian Jakobs, Johannes Seidel, Dominik Jungkenn, Stefan Mathias, Mirko Cinchetti, Martin Aeschlimann, and Claudia Felser, Topological states on the gold surface, Nat. Commun. 6, 10167 (2015).

[16] V. T. Pham, G. Zahnd, A. Marty, W. Savero Torres, M. Jamet, P. Noël, L. Vila, and J. P. Attané, Electrical detection of magnetic domain walls by inverse and direct spin hall effect, Appl. Phys. Lett. 109, 192401 (2016).

[17] Van Tuong Pham, Laurent Vila, Gilles Zahnd, Alain Marty, Williams Savero-Torres, Matthieu Jamet, and Jean-Philippe Attané, Ferromagnetic/nonmagneticnanostructures for the electrical measurement of the spin hall effect, Nano Lett. 16, 6755 (2016).

[18] Luqiao Liu, Ching-Tzu Chen, and J. Z. Sun, Spin hall effect tunnelling spectroscopy, Nat. Phys. 10, 561 (2014).

[19] Peng Li, Weikang Wu, Yan Wen, Chenhui Zhang, Junwei Zhang, Senfu Zhang, Zhiming Yu, Shengyuan A. Yang, A. Manchon, and Xi-xiang Zhang, Spin-momentum locking and spin-orbit torques in magnetic nano-heterojunctions composed of Weyl semimetal WTe2, Nat. Commun. 9, 3990 (2018).

[20] E. Lesne, Y. Fu, S. Oyarzun, J. C. Rojas-Sánchez, D. C. Vaz, H. Naganuma, G. Sicoli, J.-P. Attané, M. Jamet, E. Jacquet, J.-M. George, A. Barthélémy, H. Jaffrés, A. Fert,
M. Bibes, and L. Vila, Highly efficient and tunable spinto-charge conversion through Rashba coupling at oxide interfaces, Nat. Mater. 15, 1261 (2016).

[21] Qi Song, Hongrui Zhang, Tang Su, Wei Yuan, Yangyang Chen, Wenyu Xing, Jing Shi, Jirong Sun, and Wei Han, Observation of inverse Edelstein effect in Rashba-split 2DEG between $\mathrm{SrTiO}_{3}$ and $\mathrm{LaAlO} 3$ at room temperature, Sci. Adv. 3, e1602312 (2017).

[22] T. Nan, T. J. Anderson, J. Gibbons, K. Hwang, N. Campbell, H. Zhou, Y. Q. Dong, G. Y. Kim, N. Reynolds, X. J. Wang, N. X. Sun, S. Y. Choi, M. S. Rzchowski, Yong Baek Kim, D. C. Ralph, and C. B. Eom, Anisotropic spin-orbit torque generation in epitaxialsriro3 by symmetry design, arXiv:1808.06650 [cond-mat.mtrl-sci] (2018).

[23] P. R. Hammar and Mark Johnson, Potentiometric measurements of the spin-split subbands in a two-dimensional electron gas, Phys. Rev. B 61, 7207 (2000).

[24] Youn Ho Park, Hyun Cheol Jang, Hyun Cheol Koo, Hyungjun Kim, Joonyeon Chang, Suk Hee Han, and Heon-Jin Choi, Observation of gate-controlled spinor-bit interaction using a ferromagnetic detector, J. Appl. Phys. 111, 7 (2012).

[25] Robert H. Silsbee, Spin-orbit induced coupling of charge current and spin polarization, J. Phys. Condens. Matter 16, R179 (2004).

[26] J.-H. Lee, H.-J. Kim, J. Chang, S. H. Han, H.-C. Koo, S. Sayed, S. Hong, and S. Datta, Multi-terminal spin valve in a strong Rashba channel exhibiting three resistance states, Sci. Rep. 8, 3397 (2018).

[27] Sebastian Tölle, Ulrich Eckern, and Cosimo Gorini, Spincharge coupled dynamics driven by a time-dependent magnetization, Phys. Rev. B 95, 115404 (2017).

[28] Xin Liu and Jairo Sinova, Unified theory of spin dynamics in a two-dimensional electron gas with arbitrary spin-orbit coupling strength at finite temperature, Phys. Rev. B 86, 174301 (2012).

[29] Xin Liu and Jairo Sinova, Reading Charge Transport from the Spin Dynamics on the Surface of a Topological Insulator, Phys. Rev. Lett. 111, 166801 (2013).

[30] E. G. Mishchenko, A. V. Shytov, and B. I. Halperin, Spin Current and Polarization in Impure Two-Dimensional Electron Systems with Spin-Orbit Coupling, Phys. Rev. Lett. 93, 226602 (2004).

[31] A. A. Burkov, Alvaro S. Núñez, and A. H. MacDonald, Theory of spin-charge-coupled transport in a twodimensional electron gas with Rashba spin-orbit interactions, Phys. Rev. B 70, 155308 (2004).

[32] A. A. Burkov and D. G. Hawthorn, Spin and Charge Transport on the Surface of a Topological Insulator, Phys. Rev. Lett. 105, 066802 (2010).

[33] A. N. M. Zainuddin, S. Hong, L. Siddiqui, S. Srinivasan, and S. Datta, Voltage-controlled spin precession, Phys. Rev. B 84, 165306 (2011).

[34] Seokmin Hong, Vinh Diep, Supriyo Datta, and Yong P. Chen, Modeling potentiometric measurements in topological insulators including parallel channels, Phys. Rev. B 86, 085131 (2012).

[35] Yaroslav Tserkovnyak and Scott A. Bender, Spin hall phenomenology of magnetic dynamics, Phys. Rev. B 90, 014428 (2014). 
[36] Yan-Ting Chen, Saburo Takahashi, Hiroyasu Nakayama, Matthias Althammer, Sebastian T. B. Goennenwein, Eiji Saitoh, and Gerrit E. W. Bauer, Theory of spin hall magnetoresistance, Phys. Rev. B 87, 144411 (2013).

[37] Kerem Yunus Camsari, Samiran Ganguly, Shehrin Sayed, and Supriyo Datta, Modular approach to spintronics (2013), https://nanohub.org/groups/spintronics.

[38] Kerem Yunus Camsari, Samiran Ganguly, and Supriyo Datta, Modular approach to spintronics, Sci. Rep. 5, 10571 (2015).

[39] Shehrin Sayed, Vinh Q. Diep, Kerem Yunus Camsari, and Supriyo Datta, Spin funneling for enhanced spin injection into ferromagnets, Sci. Rep. 6, 28868 (2016).

[40] J. C. Rojas Sánchez, L. Vila, G. Desfonds, S. Gambarelli, J. P. Attané, J. M. De Teresa, C. Magén, and A. Fert, Spin-to-charge conversion using Rashba coupling at the interface between non-magnetic materials, Nat. Commun. 4, 2944 (2016).

[41] Miren Isasa, M. Carmen Martńez-Velarte, Estitxu Villamor, César Magén, Luis Morellón, José M. De Teresa, M. Ricardo Ibarra, Giovanni Vignale, Evgueni V. Chulkov, Eugene E. Krasovskii, Luis E. Hueso, Fèlix Casanova, Origin of inverse Rashba-Edelstein effect detected at the $\mathrm{Cu} / \mathrm{Bi}$ interface using lateral spin valves, Phys. Rev. B 93, 014420 (2016).

[42] Hailong Wang, James Kally, Joon Sue Lee, Tao Liu, Houchen Chang, Danielle Reifsnyder Hickey, K. Andre Mkhoyan, Mingzhong Wu, Anthony Richardella, and Nitin Samarth, Surface-State-Dominated Spin-Charge Current Conversion in Topological-Insulator-FerromagneticInsulator Heterostructures, Phys. Rev. Lett. 117, 076601 (2016).

[43] A. De Martino, R. Egger, K. Hallberg, and C. A. Balseiro, Spin-Orbit Coupling and Electron Spin Resonance Theory for Carbon Nanotubes, Phys. Rev. Lett. 88, 206402 (2002).

[44] Alessio Calzona, Matteo Carrega, Giacomo Dolcetto, and Maura Sassetti, Time-resolved pure spin fractionalization and spin-charge separation in helical luttinger liquid based devices, Phys. Rev. B 92, 195414 (2015).

[45] T. Stauber, G. Gómez-Santos, and L. Brey, Spin-charge separation of plasmonic excitations in thin topological insulators, Phys. Rev. B 88, 205427 (2013).

[46] A. V. Moroz, K. V. Samokhin, and C. H. W. Barnes, SpinOrbit Coupling in Interacting Quasi-One-Dimensional Electron Systems, Phys. Rev. Lett. 84, 4164 (2000).

[47] Bertrand I. Halperin, Spin-charge separation, tunneling, and spin transport in one-dimensional metals, J. Appl. Phys. 101, 081601 (2007).

[48] Marco Polini and Giovanni Vignale, Spin Drag and SpinCharge Separation in Cold Fermi Gases, Phys. Rev. Lett. 98, 266403 (2007).

[49] Alexander Schroer, Bernd Braunecker, Alfredo Levy Yeyati, and Patrik Recher, Detection of Spin Entanglement via
Spin-Charge Separation in Crossed Tomonaga-Luttinger Liquids, Phys. Rev. Lett. 113, 266401 (2014).

[50] P. J. Burke, Luttinger liquid theory as a model of the gigahertz electrical properties of carbon nanotubes, IEEE Trans. Nanotechnol. 1, 129 (2002).

[51] T. Valet and A. Fert, Theory of the perpendicular magnetoresistance in magnetic multilayers, Phys. Rev. B 48, 7099 (1993).

[52] Shehrin Sayed, Seokmin Hong, and Supriyo Datta, Multiterminal spin valve on channels with spin-momentum locking, Sci. Rep. 6, 35658 (2016).

[53] Seokmin Hong, Shehrin Sayed, and Supriyo Datta, Spin circuit model for $2 \mathrm{~d}$ channels with spin-orbit coupling, Sci. Rep. 6, 20325 (2016).

[54] Pengke Li and Ian Appelbaum, Interpreting currentinduced spin polarization in topological insulator surface states, Phys. Rev. B 93, 220404 (2016).

[55] Paul M. Haney, Hyun-Woo Lee, and Kyung-Jin Lee, Current induced torques and interfacial spin-orbit coupling: Semiclassical modeling, Phys. Rev. B 87, 174411 (2013).

[56] Jairo Sinova, Sergio O. Valenzuela, J. Wunderlich, C. H. Back, and T. Jungwirth, Spin hall effects, Rev. Mod. Phys. 87, 1213 (2015).

[57] A. Hoffmann, Spin hall effects in metals, IEEE Trans. Magn. 49, 5172 (2013).

[58] H. L. Wang, C. H. Du, Y. Pu, R. Adur, P. C. Hammel, and F. Y. Yang, Scaling of Spin Hall Angle in 3d, 4d, and 5d Metals from $\mathrm{Y}_{3} \mathrm{Fe}_{5} \mathrm{O}_{1} 2$ /Metal Spin Pumping, Phys. Rev. Lett. 112, 197201 (2014).

[59] H. J. Zhang, S. Yamamoto, Y. Fukaya, M. Maekawa, H. Li, A. Kawasuso, T. Seki, E. Saitoh, and K. Takanashi, Current-induced spin polarization on metal surfaces probed by spin-polarized positron beam, Sci. Rep. 4, 4844 (2014).

[60] M. Hoesch, M. Muntwiler, V. N. Petrov, M. Hengsberger, L. Patthey, M. Shi, M. Falub, T. Greber, and J. Osterwalder, Spin structure of the shockley surface state on $A u(111)$, Phys. Rev. B 69, 241401 (2004).

[61] A. Tamai, W. Meevasana, P. D. C. King, C. W. Nicholson, A. de la Torre, E. Rozbicki, and F. Baumberger, Spin-orbit splitting of the Shockley surface state on $\mathrm{Cu}(111)$, Phys. Rev. B 87, 075113 (2013).

[62] P. J. Burke, An RF circuit model for carbon nanotubes, IEEE Trans. Nanotechnol. 2, 55 (2003).

[63] S. Salahuddin, M. Lundstrom, and S. Datta, Transport effects on signal propagation in quantum wires, IEEE Trans. Electron Devices 52, 1734 (2005).

[64] Supriyo Datta, Lessons from Nanoelectronics: A New Perspective on Transport (World Scientific Publishing Co. Pte. Ltd., Singapore, 2012).

[65] N. W. Ashcroft and N. D. Mermin, Solid State Physics (Saunders, Philadelphia, PA, 1976). 